\title{
Neutron Dose and Fluence Distributions in an Infinite Air Medium
}

U.S. PARTMENT OF COMMERCE National Bureau of

Standards 



\section{Neutron Dose and Fluence Distributions in an Infinite Air Medium}

George L. Simmons and Charles M. Eisenhauer

Center for Radiation Research

Institute for Basic Standards

National Bureau of Standards

Washington, D.C. 20234

NBS Technical Notes are designed to supplement the Bureau's regular publications program. They provide a means for making available scientific data that are of transient or limited interest. Technical Notes may be listed or referred to in the open literature.

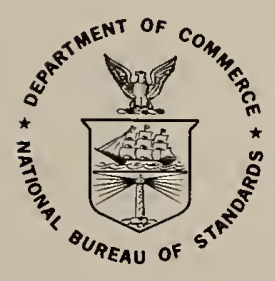

U.S. DEPARTMENT OF COMMERCE, Peter G. Peterson, Secretary

NATIONAL BUREAU OF STANDARDS, Lawrence M. Kushner, Acting Director,

Issued January 1973 


\section{National Bureau of Standards Technical Note 745}

Nat. Bur. Stand. (U.S.), Tech. Note 745,39 pages (Jan. 1973)

CODEN: NBTNAE

For sole by the Superintendent of Documents, U.S. Government Printing Office, Woshington, D.C. 20402.

Price $\$ .65$ domestic postpaid or $\$ .45$ G.P.O. Bookstore. 
Contents

Page

I. Introduction. . . . . . . . . . 1

II. Results.............. . 5

A. Benchmark Problem (Air density $1.29 \mathrm{~g} / 1$ iter) 8

B. Comparisons with $S_{n}$ Calculations (Air density

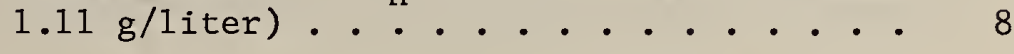

C. Simple Representations for Fluence and Dose Distribution. .......... 14

D. Angular Distributions ........ 19

III. References. ............ 34 

Neutron Dose and Fluence Distributions in an Infinite Air Medium * George L. Simmons ${ }^{* *}$ and Charles Eisenhauer

The moments method is applied to the problem of calculating the neutron dose and fluence distributions in an infinite medium of air. These calculations are compared with Monte Carlo and Discrete Ordinates $\left(S_{n}\right)$ results. Simple parametric representations for the distributions are given which facilitate the calculation of dose and flux distributions in air with a different density.

Key words: Benchmark problems; dose distributions; moments method; neutron penetration; shielding; weapons radiation.

\section{INTRODUCTION}

The moments method for solving neutron and gamma-ray transport problems is ideally suited for obtaining simple, but accurate representations of neutron dose and fluence distributions. In this note, we present data on neutron transport in air for three point isotropic sources of interest in shielding applications. These sources are: 1) fission source, 2) $14-\mathrm{MeV}$ source, and 3) a typical thermonuclear source. These results are compared with similar data obtained using the Monte Carlo and Discrete Ordinates $\left(S_{n}\right)$ techniques. We also give simple parametric representations for the dose and fluence which may be used to calculate these quantities at various distances in air of arbitrary density.

The cross section data used in these calculations are taken from

Work supported by the Defense Civil Preparedness Agency, Washington, D.C. 20310 **

Present address: Babcock \& Wilcox, Lynchburg, Va. 24505 
the ENDF/B-I compilation for oxygen $[1]^{1}$ and nitrogen $[2]$ and are the same as those used by Straker in Shielding Benchmark Problem 3.0 [3]. Air was assumed to be $79 \%$ nitrogen and $21 \%$ oxygen by volume, yielding nuclear densities of $4.25 \times 10^{-5}$ and $1.13 \times 10^{-5}$ nuclei/barm-cm, respectively, for an air density of 1.29 grams/1iter. Our calculations require that some prescription be given for interpolating on the basic cross section data. We used the specification given on the ENDF/B data file, namely, linear log-log interpolation on the cross sections and linear interpolation on the Legendre expansion coefficients of the elastic scattering angular distribution.

Calculation of spatial distributions by the moments method involves two steps. First, spatial angular moments of the fluence are obtained. These moments are then used to reconstruct distributions at various depths. We used the computer code MOMENT-I [4] to calculate spatialangular moments at 510 energies between $15 \mathrm{MeV}$ and $10^{-4} \mathrm{eV}$. The solution grid used in these calculations is given in Table I. A11 of the source

Table I. Solution Energy Grid Specification

\begin{tabular}{ccr} 
Upper & \multicolumn{2}{c}{ Intervals } \\
Energy (MeV) & Lethargy & Number \\
\hline 15.0 & 0.0314 & 35 \\
5.0 & 0.0196 & 200 \\
0.1 & 0.0316 & 60 \\
0.015 & 0.0769 & 125 \\
$1.0 \times 10^{-6}$ & 0.1023 & 90 \\
$1.0 \times 10^{-10}$ & & \\
\hline
\end{tabular}

spectra used in these calculations were normalized to one neutron emitted. The fission source energy distribution used has the form

\footnotetext{
1 Figures in brackets indicate the literature references at the end of this paper.
} 


$$
S(E)=0.453 \operatorname{Sinh}(\overline{\sqrt{2.29 E}}) \exp (-E / 0.965)
$$

The $14 \mathrm{MeV}$ source was assumed to be represented by a histogram between 15 and $12.2 \mathrm{MeV}$. The thermonuclear weapon spectrum is given by Straker [5] and was also represented in histogram form.

In obtaining the fluence from the moments, we could perform reconstructions at all 510 energies. However, this would produce more data than can be easily managed. Also, since many of the calculational tools used in shielding analysis produce multigroup results, it is desirable to give our results in a comparable form. For these reasons, we have found it convenient to define multigroup moments as

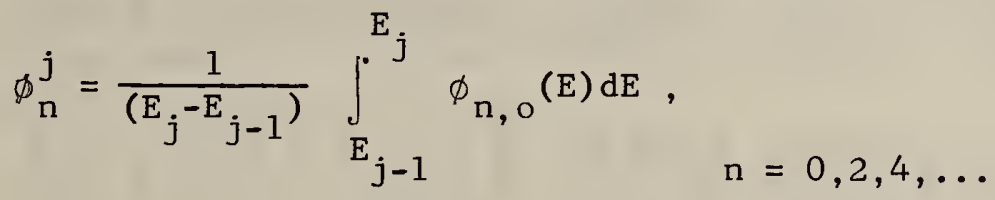

where $E_{j}$ and $E_{j-1}$ are the group boundaries for the $j \frac{\text { th }}{j}$ energy group (see Table II). Consistent with this procedure, we can weight these multigroup moments by a dose response functions, $C_{j}$, and sum over all groups:

$$
D_{n}=\sum_{i=1}^{22} \Delta E_{j} \phi_{n}^{j} C_{j}
$$

thereby obtaining dose moments (See Table II for a set of dose response functions for several detectors of interest). The dose moments can then be used to reconstruct dose distributions.

We have applied two different procedures in reconstructing distributions from moments: 1) function fitting and 2) plural series approxi- 


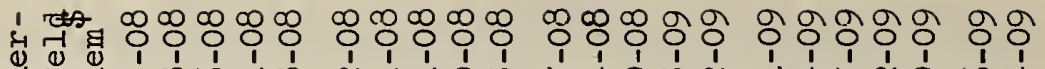

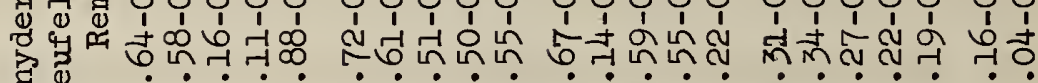

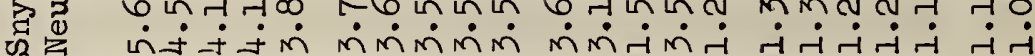

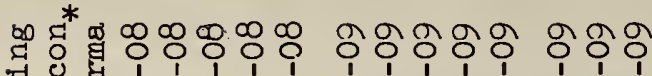

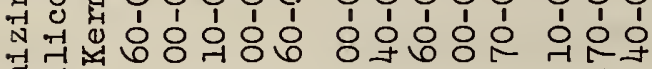

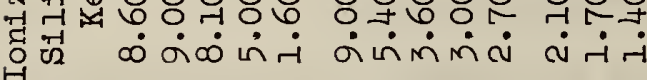

द ध :

先 건

ठี

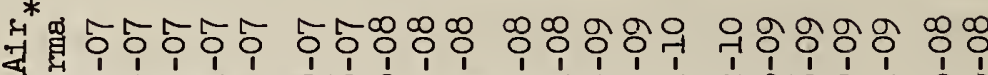

@

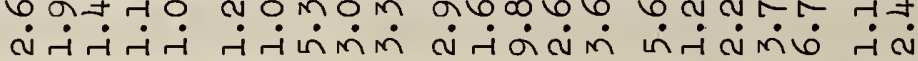

*

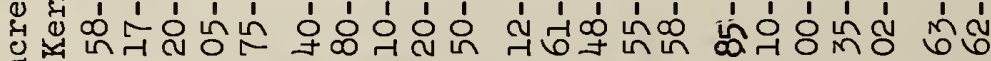

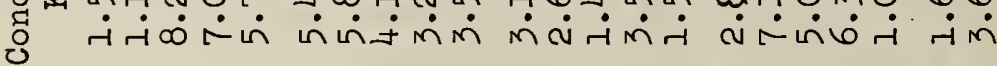

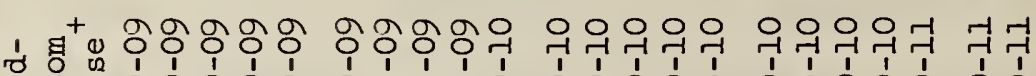

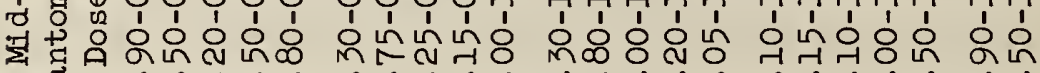

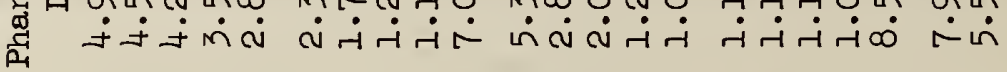

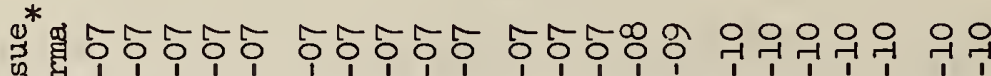
की

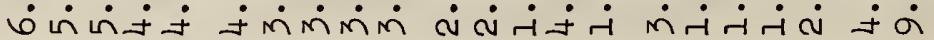

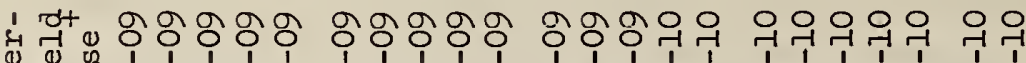
dैษ 远

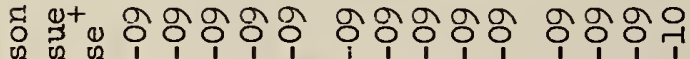

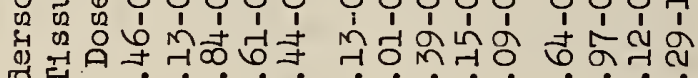

進

4

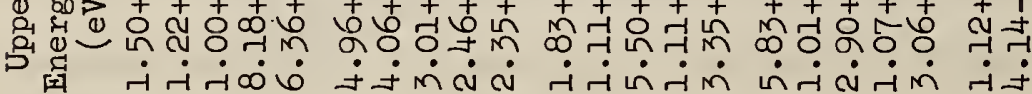

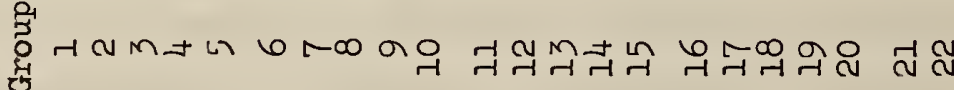


mations [6]. The first procedure has the advantage that the representations obtained for the distributions are relatively simple and are therefore easily used in point-kernel type calculations. The plural series technique, on the other hand, has the advantage that it enables us to estimate the truncation error associated with using only a finite number of moments in reconstructing the distribution. The function fitting representations make use of the form

$$
4 \pi R^{2} D(R)=D_{0} e^{-\sum_{0} R}+\sum_{i=1}^{N} \alpha_{i}\left(\sum_{0} R / \beta_{i}\right) e^{-\sum_{0} R / \beta_{i}}
$$

while the plural series representation uses the product of exponentials and U-polynomials [6] and has the form

$$
4 \pi R^{2} D(R)=D_{0} e^{-\sum{ }_{0} R}+\left(\sum_{0} R\right)^{k} \sum_{j=1}^{3} A_{j} / X_{j}^{k+1} \sum_{i=1}^{10} a_{i j} U_{n}^{k}\left(\sum R_{0} / X_{j}\right) e^{-\sum_{0} R / X_{j}}
$$

Here $\Sigma_{0}$ is the mean-free-path for neutrons at the highest energy considered, i.e. $15 \mathrm{MeV}$, and $k$ is a parameter which is specified for the fit and is usually set equal to unity. In both cases, $D_{0}$ is the value of $4 \pi R^{2}$ times the unscattered flux or dose at $R=0 . D_{0}$ is easily calculated from Equations (2) and (3), provided that the source energy distribution, $S(E)$ is substituted for the moments, $\phi_{n o}(E)$.

\section{RESULTS}

We first compare our results with existing calculations. Theoretically, in the reconstruction of distributions from moments, an infinite 
number of moments are required to obtain the correct distribution. Since only a finite number of moments (six to ten) are available to characterize the distribution, we estimate the truncation error due to neglect of remaining moments.

The apparent convergence of the distribution, using successively more moments in the fit, has been used in the past to assess the truncation error. That is to say, distributions are constructed using $\mathrm{N}$ moments and $\mathrm{N}+1$ moments, and the difference between the two distributions is examined. Generally, as more moments are used, these differences become smaller. However, there is no theoretical justification for assuming that the distributions converge to the correct value.

Spencer [6] has developed independent procedures for estimating the truncation error when plural series approximations are used to represent the distribution. These procedures are based on a more rigorous theory which gives criteria guaranteeing convergence, and which gives rigorous bounds to the truncation error which involve the norm of the unknown function. We had no difficulty satisfying the convergence criteria; but as in Ref. 6, we rely on estimations rather than rigorous values for the norm and therefore also for the truncation error bounds. The truncation error bounds represent maximum errors, rather than probable errors. They are therefore a conservative estimate of the truncation error.

For the data presented in this report, we have relied heavily upon the convergence of the distribution as the method for selecting the best fit to the moments. We have then applied Spencer's approach to determine rigorous error bounds on the selected fit. In Table III, we give a typical distribution which illustrate both the convergence and error 


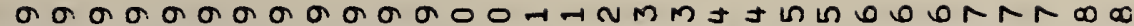
1 1 1

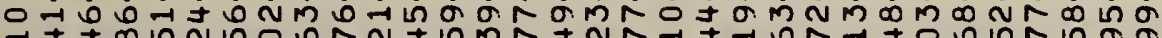

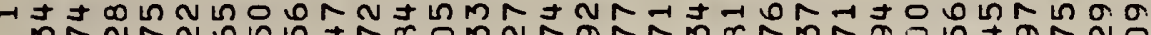
MNNRN

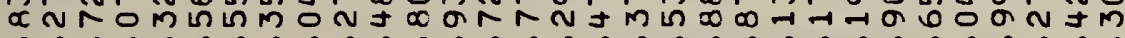

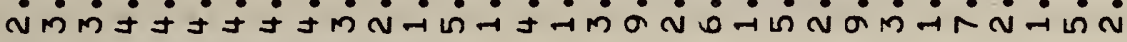

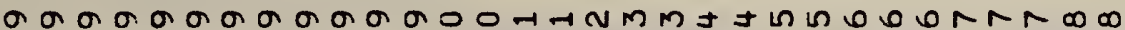

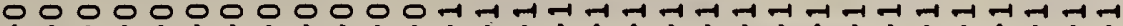

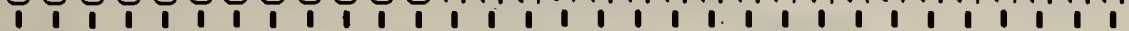

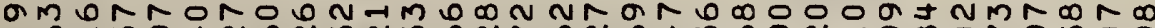

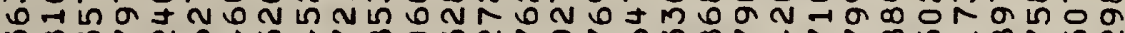
$m \infty m R N$ 约

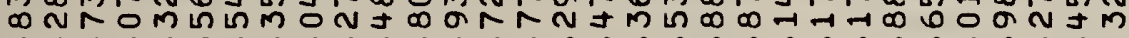

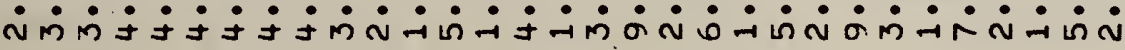

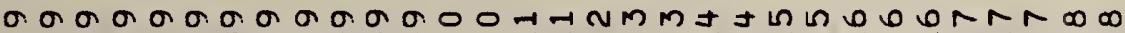

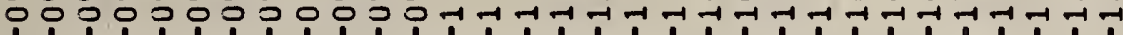

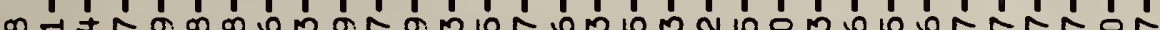

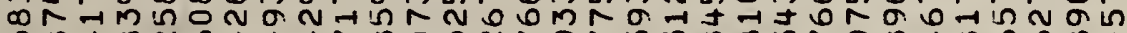

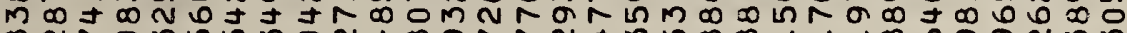
$\infty$ N ${ }_{0} m$ ?

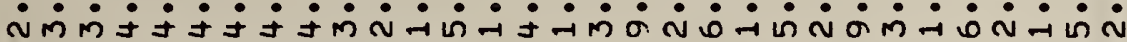

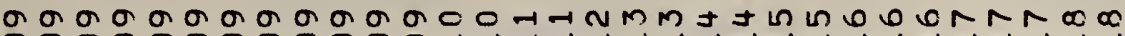
이시 N $m$ ก

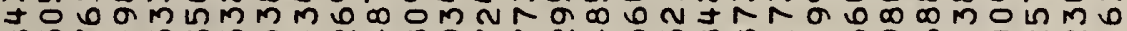
क

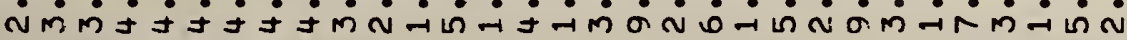

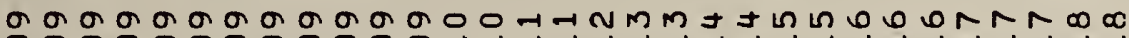

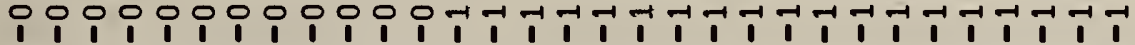
$0 \infty N$ in 0 N $\infty$ in NoR-7fJ

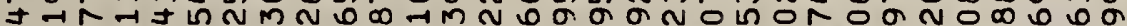
$\infty m R-m$ n

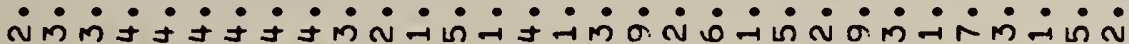

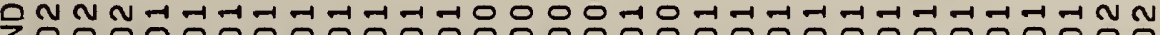

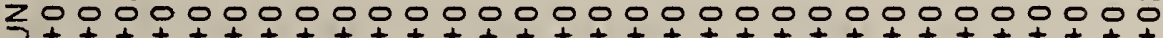

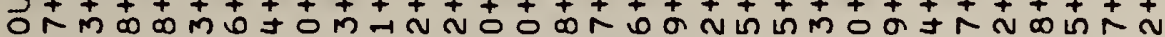

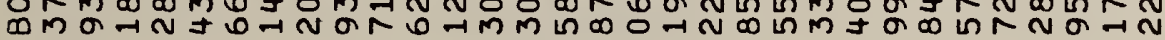

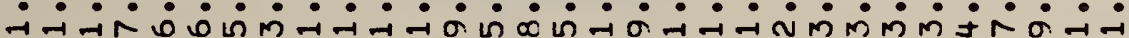

0ㅇㅇㅇㅓ-1 -

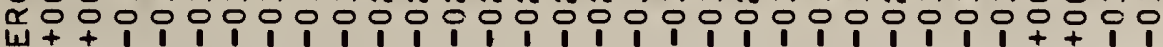

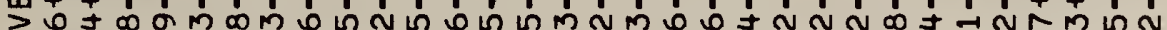

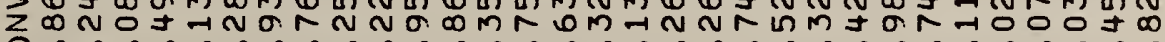

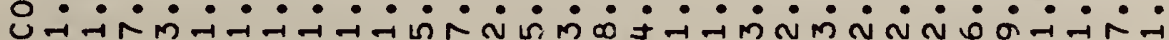

n n

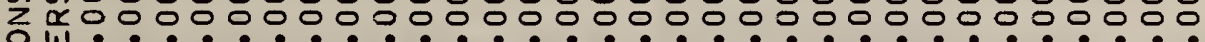
ow " $\sum_{m}^{\infty} \sum_{-1} N$ N $x$

Fogar UL

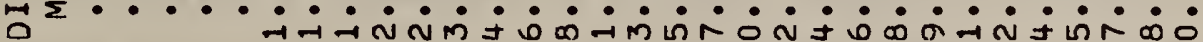

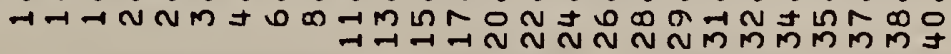

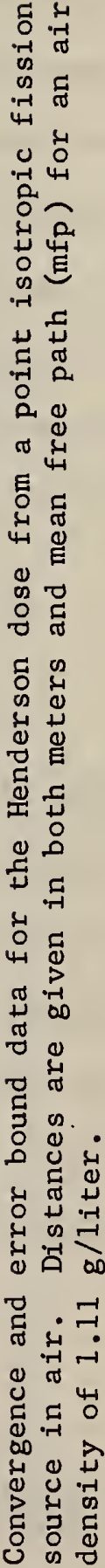

H 
bound data for the distribution. Under the heading CONVERG, the percentage differences between an 8 and 10 moment $\mathrm{fit}$ is given. Note that the truncation error bound (BOUND) calculated as indicated in References [6] and [7], is larger than the convergence indicator (CONVERG). This gives us confidence that when convergence is good, the convergence indicator is a realistic estimate of the error that can be attributed to distribution construction. However, if this indicator is large, then we must rely solely upon error bound calculations to indicate the truncation error.

\section{A. Benchmark Problem (Air density $1.29 \mathrm{~g} / 1$ iter)}

We have calculated the multigroup fluence and Henderson dose at several distances for the fission and $14 \mathrm{MeV}$ sources, and compared them with Monte Carlo calculations by Straker [3]. In Table IV, we present a comparison of the multigroup fluences for the two sources for various distances from the source point. Generally the two methods compare very favorably, except in regions where the statistical error of the Monte Carlo results is large. Table $\mathrm{V}$ shows a similar comparison for the Henderson dose.

B. Comparisons with $\mathrm{S}_{\mathrm{n}}$ Calculations (Air density $1.11 \mathrm{~g} / 1$ iter)

A large tabulation of fluences and dose has been made by Straker [5] for the three source types of interest, as well as for other source distributions. These calculations were made using the ANISN code $\left(\mathrm{S}_{16} \mathrm{P}_{5}\right)$ for a 5000 meter sphere. Figures 1, 2, and 3 give comparisons of the multigroup spectra for the three sources of interest, as calculated by the $S_{n}$ and moments methods. The major discrepancies are in the groups 


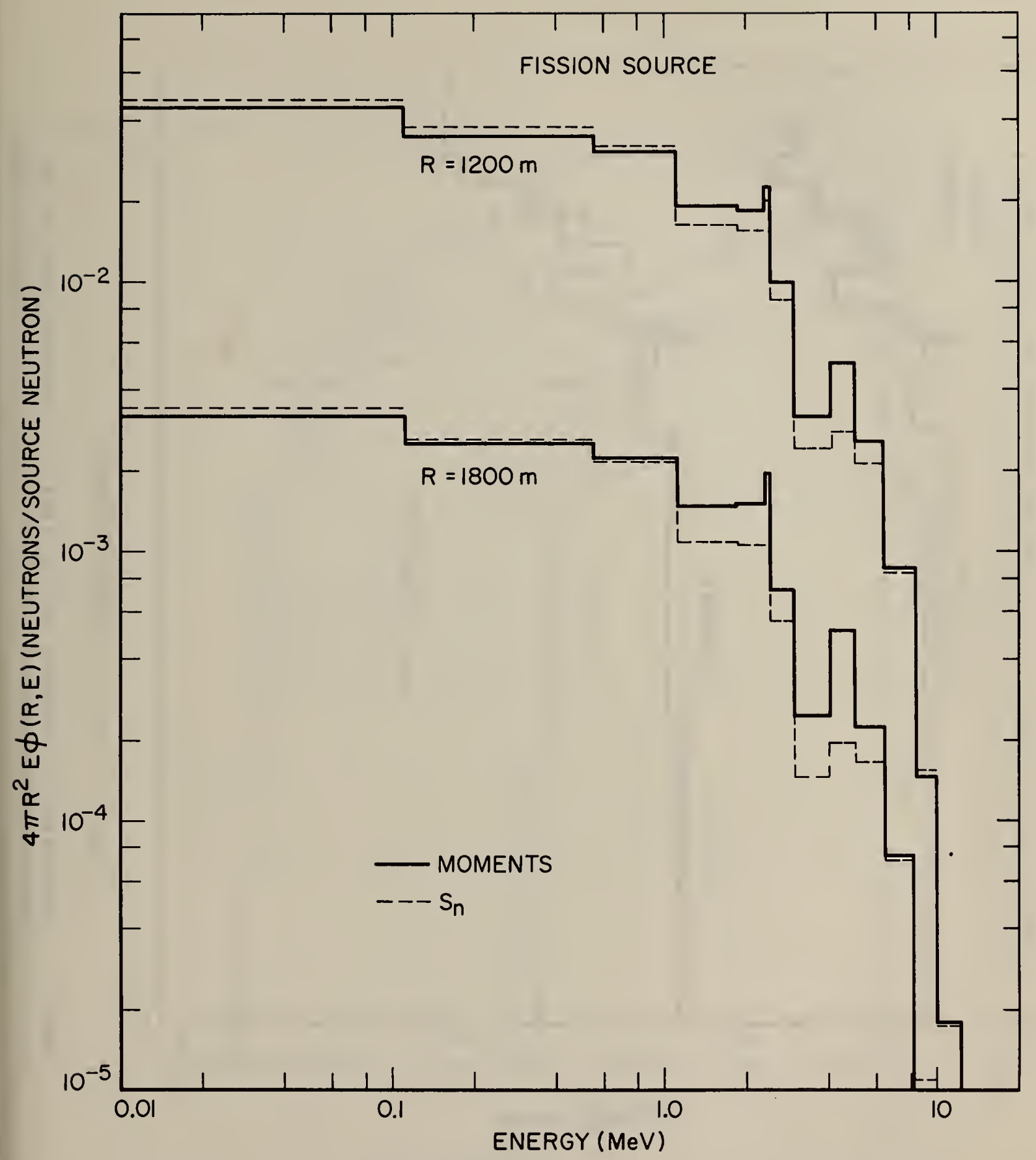

Figure 1. Comparișon of moments and $S_{n}$ (Ref. 5) calculations from a fission source at two depths in air. 


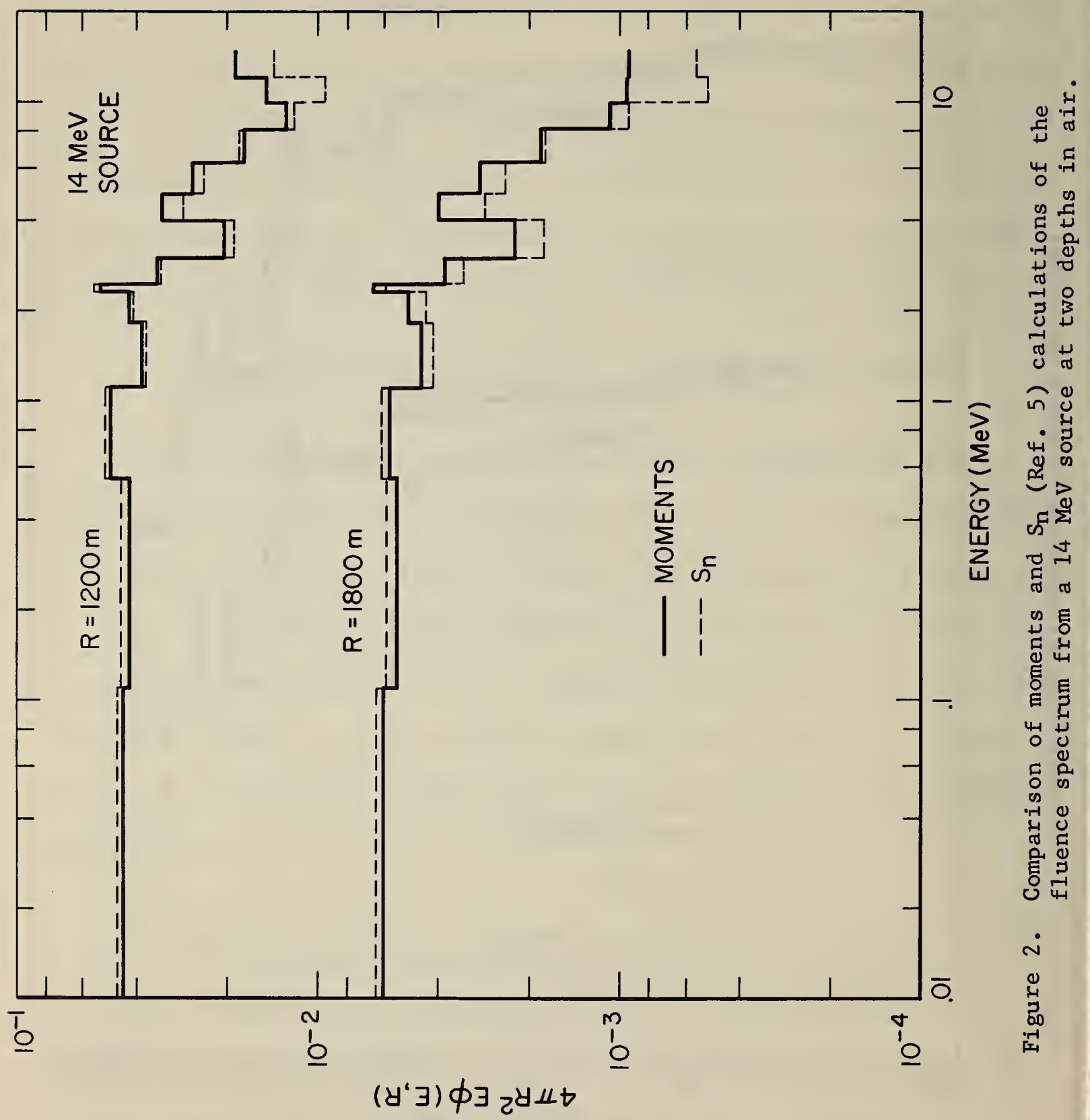




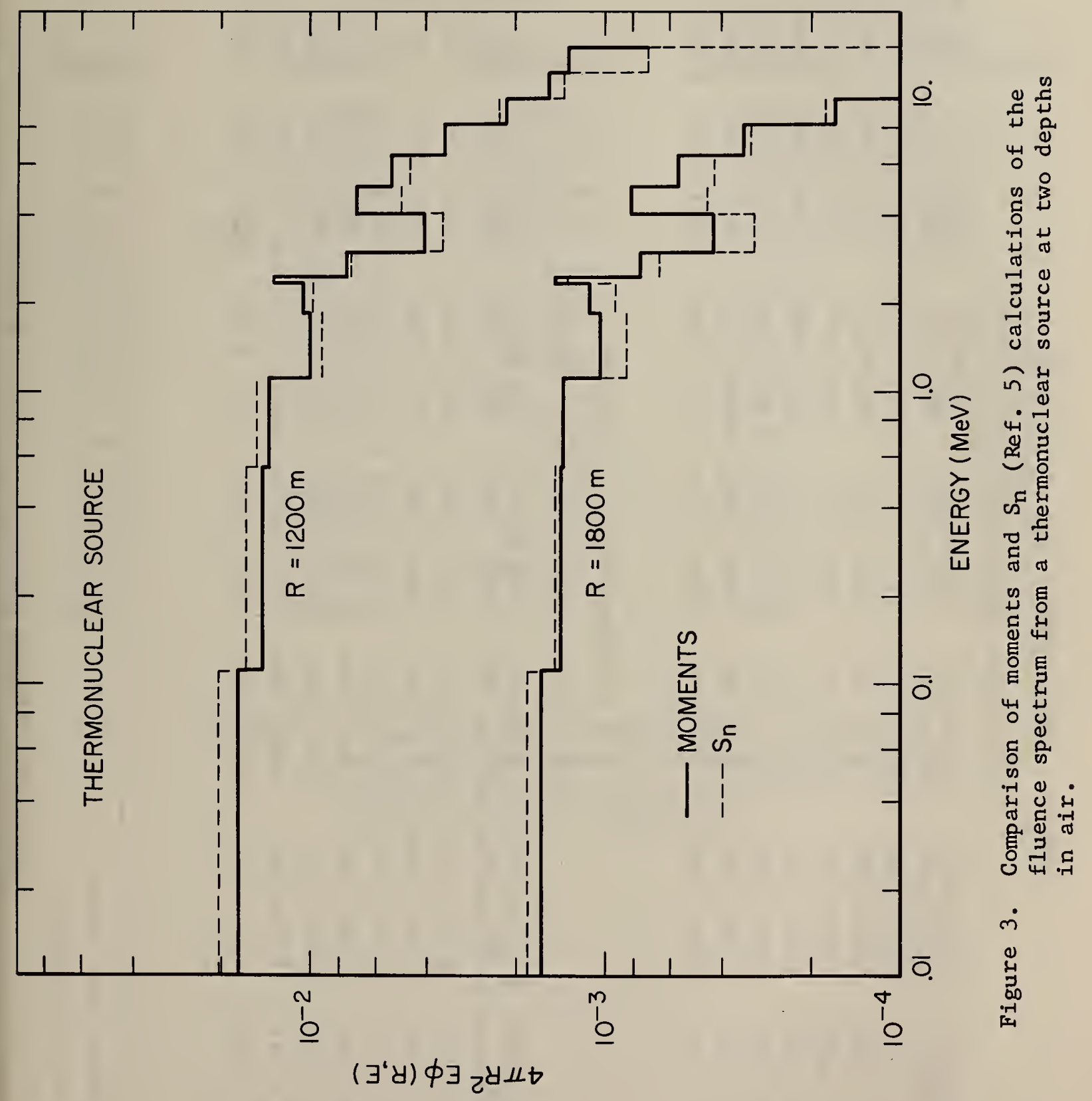



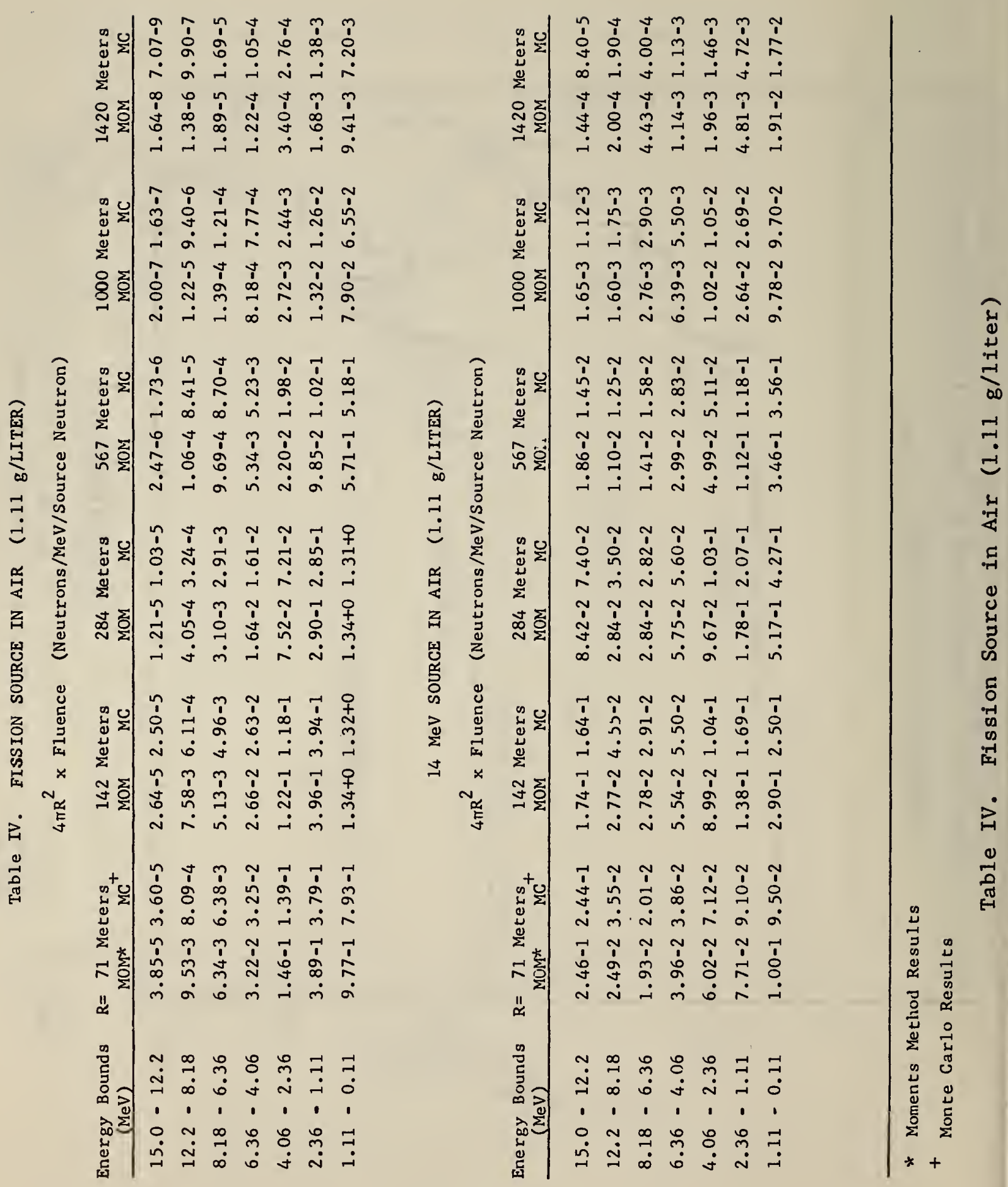
$4 \pi R^{2} \times$ Henderson Dose (Rad $-\mathrm{cm}^{2} /$ Source Neutron)

R

(meters)

71

142

284

380

472

567

662

755

850

1000

1135

1275

1420
Fission Source

Moments

4.15-09

4. 59-09

3. 83-09

2.93-09

2.14-09

1.47-09

9. $85-10$

6.49-10

4.17-10

2.03-10

1.04-10

5.17-11

2. 84-11
Monte Carlo

3.98-09

4.60-09

3.70-09

2.65-09

1.98-09

1.32-09

8.40-10

5.60-10

3. 30-10

1.75-10

7.51-11

4.25-11

2.18-11
$14 \mathrm{MeV}$ Source

Moments

5.74-09

5.60-09

5.33-09

3.72-09

2.92-09

2.19-09

1.61-09

1.16-09

8.18-10

4.58-10

2.66-10

1.48-10

7.98-10
Monte Carlo

5.97-09

6.03-09

4.75-09

3. 86-09

2.99-09

2.20-09

1.60-09

1.14-09

7. $80-10$

4.40-10

2. $42-10$

1. 38-10

7. 55-11

Table V. Comparison between Monte Carlo and moments methods calculations of Henderson dose for a point isotropic fission and $14 \mathrm{MeV}$ source in air (density $1.29 \mathrm{~g} /$ liter). 
6 and 9. At these energies there exist minima in the air cross section which may be difficult to represent in the multigroup procedure [5]. Consequently, the streaming through these minima will not be calculated correctly. This effect will become more pronounced as the sourcedetector separation is increased. In Table VI we show a comparison of the Henderson and Snyder-Neufeld dose for the three sources of interest. Generally the two calculations compare favorably, with the moments results tending to be higher than the $S_{n}$ results. We feel that this is due to the increased streaming that is predicted by the moment calculations, as well as to the leakage effect that is associated with the finite sphere used in the $s_{n}$ calculations.

\section{Simple Representations for Fluence and Dose Distribution.}

For point kernel calculations, it is useful to have a simple set of parameters which can be used to represent the dose and fluence for the three sources. Since we are interested in simple representations of these distributions, the results of the function fitting calculations are used. We note in passing that function fitting and plural series techniques give the same distribution over the range of interest. In making these tabulations, we restrict ourselves to representations of the distributions by 11 or fewer parameters. In Tables VII, VIII, and IX, we give coefficients for the dose quantities in Table II. The form used in calculating the distributions from the coefficients is

$$
4 \pi R^{2} D(R)=D_{0} e^{-\sum_{0} R}+\sum_{i=1}^{5} a_{i}\left(\sum_{0} R / \beta_{i}\right) e^{-\Sigma_{0} R / \beta_{i}}\left(\operatorname{rads}-c^{2}{ }^{2}\right)
$$


$4 \pi R^{2} \times$ Henderson Dose (Rads $-\mathrm{cm}^{2} /$ Source Neutron)

\begin{tabular}{|c|c|c|c|c|c|c|}
\hline \multirow{2}{*}{$\begin{array}{c}\mathrm{R} \\
\text { (Meters) }\end{array}$} & \multicolumn{2}{|c|}{ Fission Source } & \multicolumn{2}{|c|}{$14 \mathrm{MeV}$ Source } & \multicolumn{2}{|c|}{ Weapon Source } \\
\hline & Moments & $s_{n}$ & Moments & $s_{n}$ & Moments & \\
\hline 150 & $4.50-09$ & $4.49-09$ & $5.69-09$ & $6.07-09$ & $2.41-09$ & $2.49-09$ \\
\hline 300 & $4.05-09$ & $3.93-09$ & $4.88-09$ & $5.06-09$ & $1.95-09$ & $2.03-09$ \\
\hline 600 & $1.81-09$ & $1.76-09$ & $2.59-09$ & $2.60-09$ & $8.55-10$ & $8.85-10$ \\
\hline 900 & $5.92-10$ & $5.82-10$ & $1.09-09$ & $1.08-09$ & $3.08-10$ & $3.13-10$ \\
\hline 1200 & $1.72-10$ & $1.68-10$ & $4.04-10$ & $3.94-10$ & $1.02-10$ & $1.01-10$ \\
\hline 1800 & $1.29-11$ & $1.16-11$ & $4.48-11$ & $4 \cdot 24-11$ & $9.91-12$ & $9.12-12$ \\
\hline 2400 & $9.30-13$ & $7.19-13$ & $4.32-12$ & $3.85-12$ & & \\
\hline 3000 & $6.80-14$ & $4.27-14$ & $3.87-13$ & $3.17-13$ & & \\
\hline 3600 & $5.08-15$ & $2.52-15$ & $3.34-14$ & $2.46-14$ & & \\
\hline 4200 & $3.84-16$ & $1.49-16$ & $2.81-15$ & $1.84-15$ & & \\
\hline 4800 & $2.92-17$ & $8.43-18$ & $2.32-16$ & $1.26-16$ & & \\
\hline
\end{tabular}

$4 \pi \mathrm{R}^{2} \times$ Snyder-Neufeld Dose (Rads- $\mathrm{cm}^{2} /$ Source Neutron)

\begin{tabular}{|c|c|c|c|c|c|c|}
\hline \multirow{2}{*}{$\begin{array}{c}R \\
\text { (Meters) }\end{array}$} & \multicolumn{2}{|c|}{ Fission Source } & \multicolumn{2}{|c|}{$14 \mathrm{MeV}$ Source } & \multicolumn{2}{|c|}{ Weapon Source } \\
\hline & Moments & $s_{n}$ & Moments & $S_{n}$ & Moments & $s_{n}$ \\
\hline 150 & $6.80-09$ & $7.40-09$ & $8.40-09$ & $8.25-09$ & $5.47-09$ & $5.49-09$ \\
\hline 300 & $7.08-09$ & $7.19-09$ & $7.01-09$ & $7.22-09$ & $4.42-09$ & $4.55-09$ \\
\hline 600 & $3.82-09$ & $3.62-09$ & $4.05-09$ & $4.05-09$ & $1.76-09$ & $1.85-09$ \\
\hline 900 & $1.30-09$ & $1.28-09$ & $1.84-09$ & $1.79-09$ & $6.15-10$ & $6.39-10$ \\
\hline 1200 & $3.86-10$ & $3.82-10$ & $7.10-10$ & $6.88-10$ & $2.01-10$ & $2.02-10$ \\
\hline 1800 & $2.89-11$ & $2.70-11$ & $8.24-11$ & $7.87-11$ & $1.93-11$ & $1.84-11$ \\
\hline 2400 & $2.02-12$ & $1.66-12$ & $8.11-12$ & $7.39-12$ & & \\
\hline 3000 & $1.41-13$ & $9.78-14$ & $7.35-13$ & $6.20-13$ & & \\
\hline 3600 & $1.03-14$ & $5.68-15$ & $6.36-14$ & $4.86-14$ & & \\
\hline 4200 & $8.10-16$ & $3.31-16$ & $5.35-14$ & $3.65-14$ & & \\
\hline 4800 & $6.84-17$ & $1.83-17$ & $4.40-16$ & $2.48-16$ & & \\
\hline
\end{tabular}

Table VI. Comparison between $S_{n}$ calculations and moments method calculation of Henderson and Snyder-Neufeld dose for a point isotropic fission, $14 \mathrm{MeV}$, and thermonuclear weapon source in air (density $1.11 \mathrm{~g} / 1$ iter). $S_{\mathrm{n}}$ data taken from Reference 5. 
FISSION SOURCE IN AIR

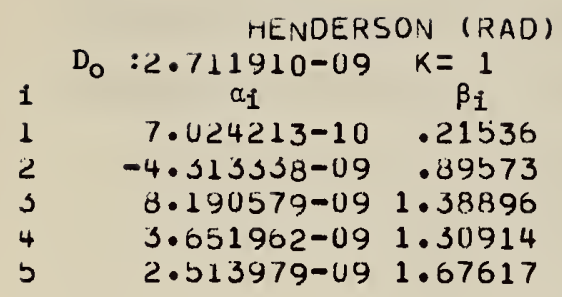

$D_{O}:$ MID-PHANTOM (RAD) 8.776196-10 $K=1$

i

1

1

$$
4.018530-09 \quad .49140
$$

$4.205045-091.47419$

-3.063073-09 .66646

$1.109593-101.85908$

0.0000001 .00000

$$
\text { NON-IUN. SILICON KERMA }
$$$$
D_{0}: 3.710731-09 \quad K=1
$$$$
\begin{array}{rr}
9.003585-10 & .69297 \\
-1.335754-08 & .98297 \\
1.402235-08 & 1.15605 \\
1.073952-08 & 1.44839 \\
2.475567-09 & 1.69788
\end{array}
$$

$$
\begin{array}{cc}
\text { SNYDER-NEUFELD } & \text { (RAD) } \\
3.843843-09 & K=1 \\
\alpha_{1} & \beta_{1} \\
3.032895-08 & .48881 \\
2.651148-08 & 1.46643 \\
-3.700481-08 & .63225 \\
6.949531-10 & 1.85179 \\
0.000000 & 1.00000
\end{array}
$$

CONCRETE KERMA (ERG/G)

3.453603-08 $K=1$

$$
a_{1}
$$

9.839580-09 .21622

$-5.994808-08 \quad .89933$

$3.863627-08 \quad 1.39454$

$1.162011-07 \quad 1.35447$

$3.363860-08 \quad 1.67387$
TISSUE KERMA (ERG/G)

2.752741-07 $K=1$

$a_{1}$

$9.387321-08 \quad .21780$

$-5.611913-07 \quad .90590$

8.124505-06 1.40472

$-6.808031-061.41258$

$3.164664-071.06805$

$\begin{array}{cc}\text { AIR KERMA } & \text { (ERG/G) } \\ 4.324899-08 & K=1 \\ \alpha_{1} & \beta_{1} \\ 9.665720-08 & .49046 \\ 1.955809-07 & 1.47138 \\ -1.497707-07 & .77937 \\ 6.125755-09 & 1.84231 \\ 0.000000 & 1.00000\end{array}$

SNYDER-NEUFELD (REM) 3.210515-08 $K=\frac{1}{\beta_{i}}$

$-3.173191-071.13018$

4.050881-07 1.25199

$5.584109-081.04183$

$0.000000 \quad 1.00000$

$0.000000 \quad 1.00000$

$$
4 \pi R^{2} \times \operatorname{Dose}(R)=D_{0} \exp \left(-\Sigma_{0} R\right)+\sum_{i=1}^{5} a_{i}\left(\Sigma_{0} R / \beta_{i}\right)^{k} \exp \left(-\Sigma_{0} R / \beta_{i}\right)
$$

Table VII. Coefficients for calculating dose distributions in air for a point isotropic fission source. The dose distributions have units of (dose units $-\mathrm{cm}^{2}$ per source neutron). $\Sigma_{0}^{-1}$ is 133.059 meters for an air density of $1.11 \mathrm{~g} / 1$ iter. 
$14 \mathrm{MeV}$ SOURCE IN AIR

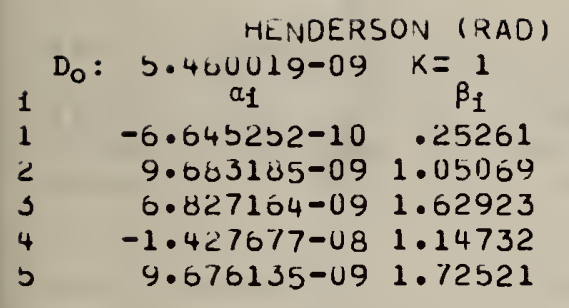

$\begin{array}{cc}\text { SNYDER-NEUFELD } & \text { (RAD) } \\ 7.000024-09 & K= \\ \alpha_{i} & \beta_{i} \\ -8.955006-09 & .15094 \\ 7.164796-09 & .69763 \\ 4.898157-08 & 1.34196 \\ 2.860304-08 & 1.70389 \\ -6.504560-08 & 1.31416\end{array}$

$$
\begin{aligned}
& \text { MID-PHANTOM (RAD) } \\
& D_{0}: \text { 4.900017-09 } K=1 \\
& \begin{array}{rr}
-4 \cdot 143389-10 & .23546 \\
1 \cdot 121504-09 & .97933 \\
3.092354-09 & 1.31858 \\
-2.612731-09 & 1.26748 \\
4 \cdot 960820-09 & 1.71004
\end{array}
\end{aligned}
$$

NUN-IUN. SILICON KERMA

$$
D_{0}: 7.500026-09 \quad k=1
$$

$2.734338-08 \quad 1.63360$

$-1.368610-08 \quad 1.40440$

$1.971405-091.86455$

$-5.864663-12 \quad 2.35862$

0.0000001 .00000

$$
\begin{array}{cc}
\text { CONCRETE KERMA } & \text { (ERG/G) } \\
1.580005-07 & K=1 \\
\alpha_{i} & \beta_{i} \\
-1.517908-08 & .23996 \\
6.707914-08 & .99806 \\
8.164871-08 & 1.54762 \\
-9.334668-08 & 1.16231 \\
1.620361-07 & 1.71573
\end{array}
$$

ION. SILICON KERMA 8.600030-08 $K=1$ $a_{i}$

$\beta_{1}$

$.6 .418379-09 \quad .21030$

$7.669295-09 \quad .87471$

4.430919-08 1.35636

3.612633-08 1.59763

7.979537-09 1.74196
TISSUE KFRMA (EKG/G)

6.360022-07 $K=1$

$a_{i}$

$-7.657200-08 \cdot 25311$

$1.172000-00 \quad 1.05276$

8.068461-07 1.03244

$-1.694500-061.15221$

$1.003330-06 \quad 1.72744$

$$
\begin{array}{cc}
\text { AIR KERMA } & \text { (ERG/G) } \\
2.660009-07 & K=1 \\
a_{i} & \beta_{i} \\
-2.829879-08 & .23338 \\
7.271133-08 & .97071 \\
1.485257-07 & 1.50521 \\
-1.071440-07 & 1.31727 \\
2.252018-07 & 1.71239
\end{array}
$$

\section{SNYDER-NEUFELO (REM)}

5.640019-0̈8 $K=1$

$a_{i}$

$\beta_{i}$

$-9.282823-09 \quad .26209$

4.560220-07 1.09012

2.022771-07 1.09037

$-5.393797-071.13266$

$1.088691-08 \quad 1.01020$

$$
4 \pi R^{2} \times \operatorname{Dose}(R)=D_{0} \exp \left(-\Sigma_{0} R\right)+\sum_{1=1}^{5} a_{i}\left(\Sigma_{0} R / \beta_{1}\right)^{k} \exp \left(-\Sigma_{0} R / \beta_{1}\right)
$$

Table VIII. Coefficients for calculating dose distributions in air for a point isotropic $14 \mathrm{MeV}$ source. The dose distributions have units of (dose units-cm 2 per source neutron). $\Sigma_{0}^{-1}$ is 133.059 meters for an air density of $1.11 \mathrm{~g} / 1$ iter. 
THERMONUCLEAR SOURCE IN AIR

\begin{tabular}{|c|c|c|}
\hline & HENDER & $(R A D)$ \\
\hline$D_{0}:$ & $\begin{array}{c}1.598693-09 \\
a_{i}\end{array}$ & $k=\underset{\beta_{i}}{1}$ \\
\hline 1 & $2 \cdot 235079-10$ & .53182 \\
\hline 2 & $2.229882-09$ & 1.35445 \\
\hline 3 & $2.699256-09$ & 1.69711 \\
\hline 4 & $2.951245-10$ & 1.75437 \\
\hline b & U. U00000 & 1.00000 \\
\hline
\end{tabular}

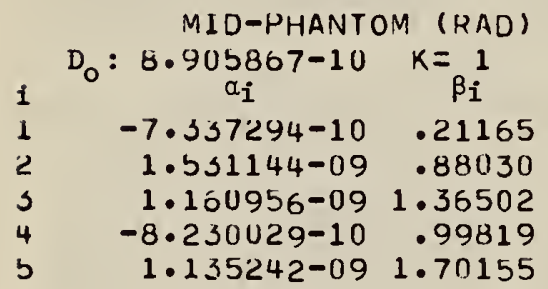

$$
\begin{array}{cc}
\text { SNYDER-NEUFELD } & (R A D) \\
2.462538-09 & K=1 \\
\alpha_{i} & \beta_{i} \\
5.087558-09 & 1.37554 \\
-8.094390-09 & .47979 \\
9.294106-09 & .63088 \\
9.040126-11 & 1.60195 \\
5.399257-09 & 1.70926
\end{array}
$$

CONCRETE KERYA (ERG/G)

$$
\begin{array}{cc}
2.863221-08 & K={ }_{\beta_{i}}^{1} \\
4.211789-09 & .61293 \\
3.432219-08 & 1.35017 \\
3.735728-08 & 1.69671 \\
2.190751-09 & 1.77446 \\
0.000000 & 1.00000
\end{array}
$$

$$
\begin{aligned}
& \text { TISSUE KERMA (EKG/G) } \\
& 1.77218 \cap-07 \quad K=1 \\
& \alpha_{i}
\end{aligned}
$$

\footnotetext{
NON-IUN. SILICON KERMA $D_{0}$ : 2.104689-09 $K=1$ $\alpha_{1}$ $\beta_{i}$ $-2.849184-09 \quad .48294$ $1.378367-09 \quad 1.44882$ $2.592974-09 \quad .43650$ $1.771836-09 \quad 1.28652$ $3.855546-091.70903$

ION. SILICON KERMA $1.139916-08 \quad K=\beta_{i}^{1}$ $-1.102444-10 \quad 1.35895$ $-3.306599-12.40243$ $3.814736-09 \quad 1.17663$ $7.002446-09 \quad 1.45429$ $4.738565-09 \quad 1.68985$ $4 \pi R^{2} \times \operatorname{Dose}(R)=D_{0} \exp \left(-\Sigma_{0} R\right)+\sum_{i=1}^{5} \alpha_{i}\left(\Sigma_{0} R / \beta_{i}\right)^{k} \exp \left(-\Sigma_{0} R / \beta_{i}\right)$
}

AIR KERMA (ERG/G)

$4.131295-08 \quad K={ }_{\beta_{i}} 1$

4.733615-08 1.39377

$-7.026025-08 \cdot 37382$

$7.407690-08 \quad .82382$

$4.939778-08 \quad 1.70684$

$0.000000 \quad 1.00000$

Table IX. Coefficients for calculating dose distributions in air for a PTI thermonuclear weapon source. The dose distributions have units of (dose units- $\mathrm{cm}^{2}$ per source neutron). $\Sigma_{0}^{-1}$ is 133.059 meters for an air density of $1.11 \mathrm{~g} / 1$ iter. 
Here $1 / \Sigma_{0}=133.905$ meters which corresponds to an energy of $15 \mathrm{MeV}$ and an air density of 1.11 grams/1iter. These same coefficients may be applied to calculations at different air densities by using simple scaling procedures based on equivalent mean free paths. In Tables X, XI, and XII, the distributions obtained by using the coefficients in Tables VII, VIII, and IX are given.

In Tables XIII, XIV, and XV, the coefficients which represent the multigroup spectra for each source are given. The form for these distributions for the $j^{\text {th }}$ energy group is

$4 \pi R^{2} \phi^{j}(R)=\phi_{0}^{j} e^{-\Sigma o_{0}^{R}}+\sum_{i=1}^{5} \alpha_{i}\left(\sum_{0} R / \beta_{i}\right) e^{-\sum_{0} R / \beta_{i}}$ (neutrons/MeV/source neutron)

Tables XVI, XVII, and XVIII give the distributions obtained using these coefficients. Note that for the thermonuclear weapon source, at very low energies and close to the source, the distributions are negative. This indicates that the coefficients for these particular energies should be used with caution at distances less than one mean-free-path. Indeed in this particular region, it is extremely difficult to fit the moments and obtain suitable distributions over the entire range.

\section{Angular Distributions.}

Angular distributions for dose or fluence can also be calculated by the moments method. Generally the procedure that is used for point sources is to calculate spatial distributions for Legendre harmonic coefficients $\dot{\psi}_{\ell}(E, z)$ for values of $\ell$ up to about 8 . In theory, the angular distributions at any distance can then be calculated by perform- 


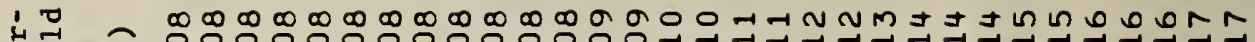

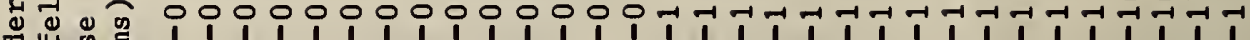
ग कृ तब

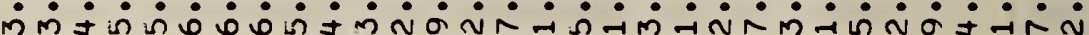

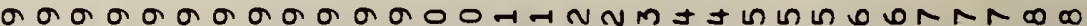

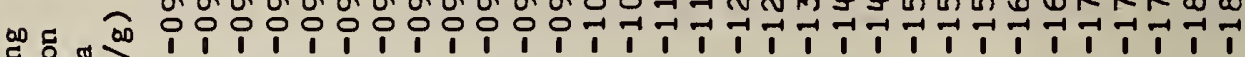

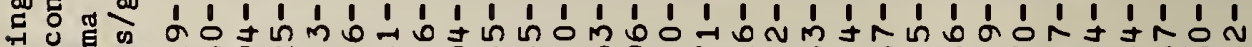

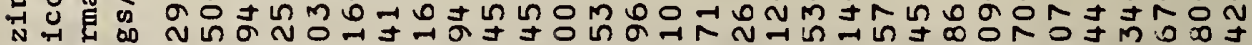

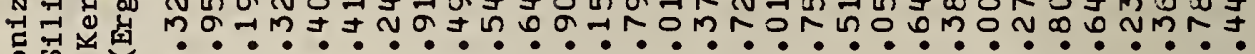

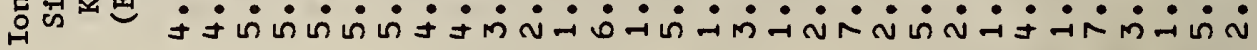

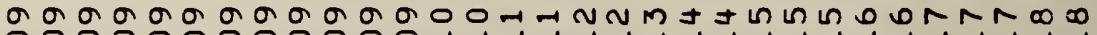

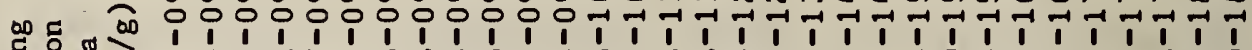
I ᄃ개 望装出

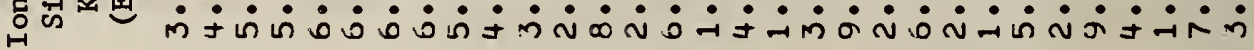

(1)

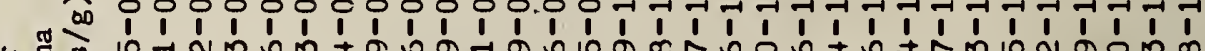
म स्य

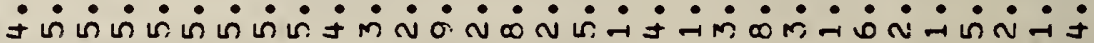

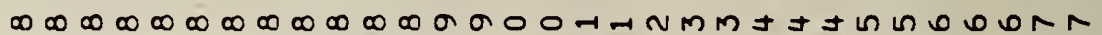

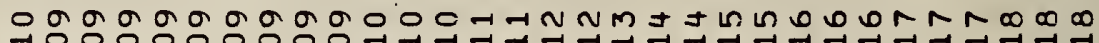

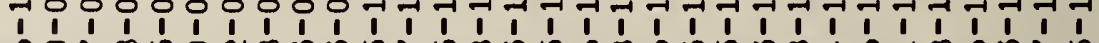

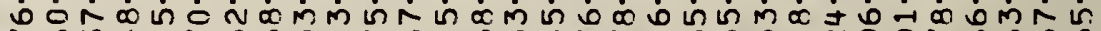

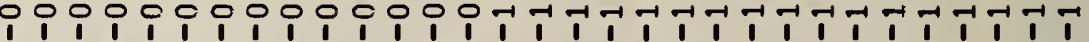
t bomRN丶

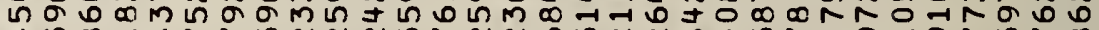

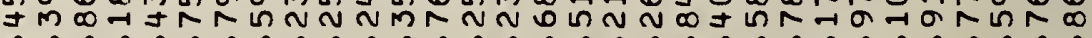

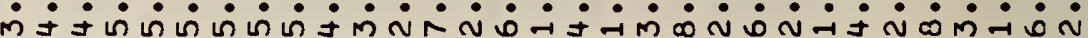

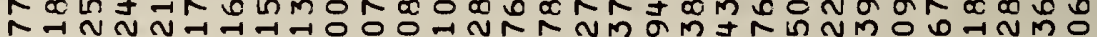

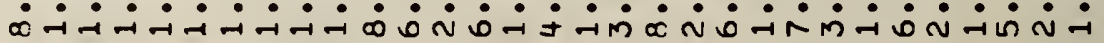

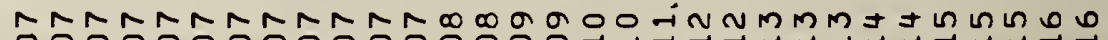

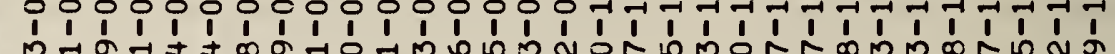

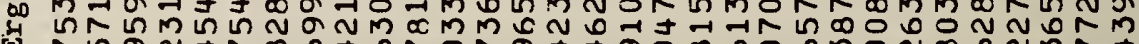

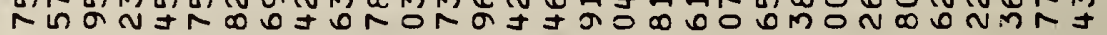

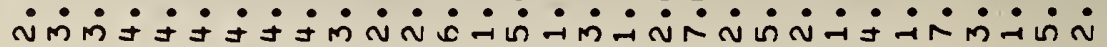

峞 눙 क兄

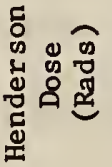
๙

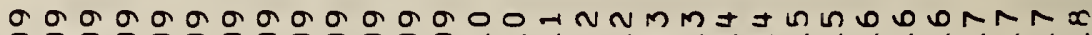

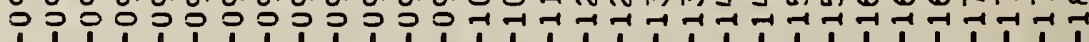

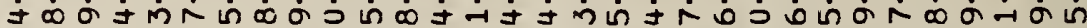

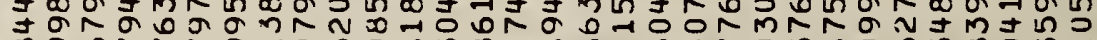
o. or

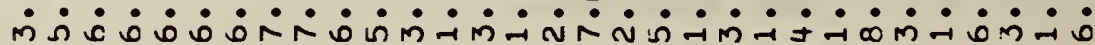

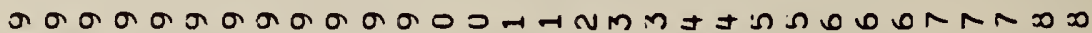

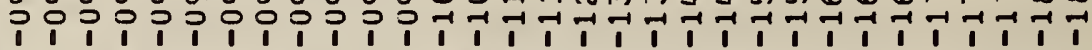

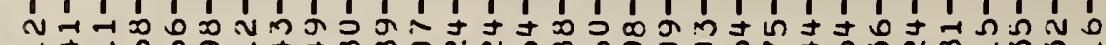

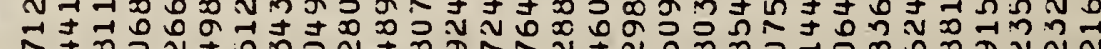
ก.

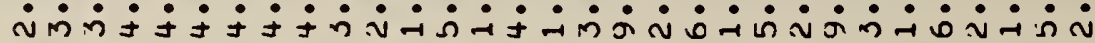


茪 प̆

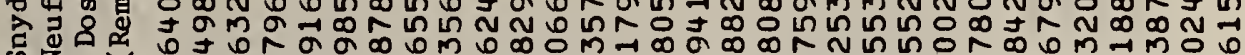

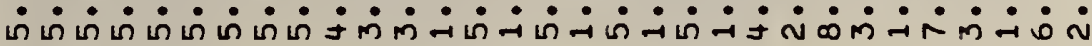

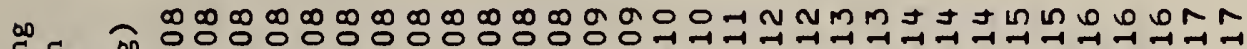
ت N H

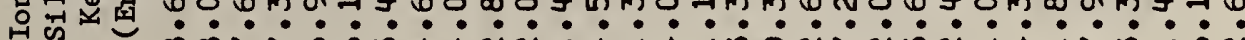

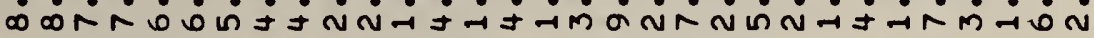

क क 1.70 ¿ 武幽 虽

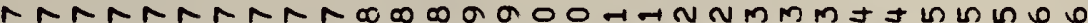

$000000000000000-1-1-1-1-1-1-1-1-1-1-1$ 4

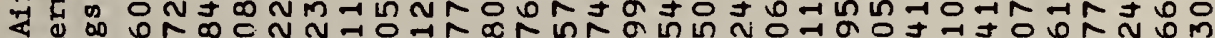
药

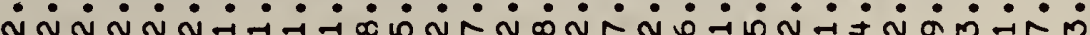

NNNNNRN

॥ क o

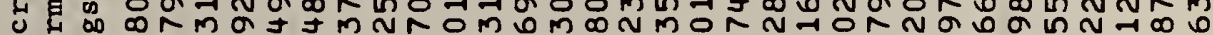

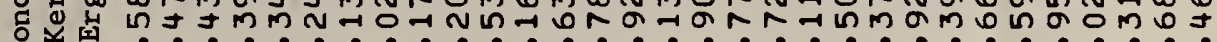

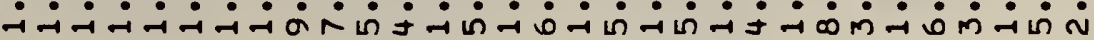

章。

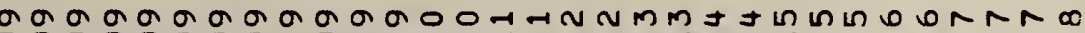

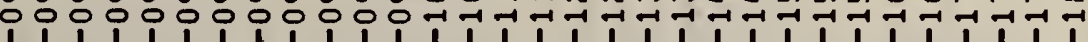

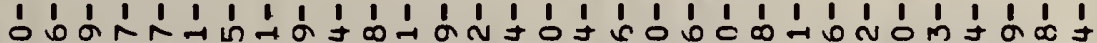

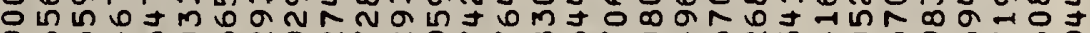
a

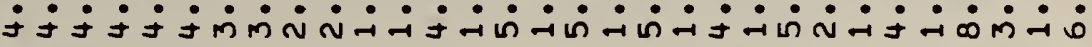

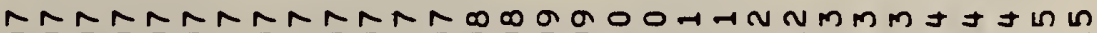

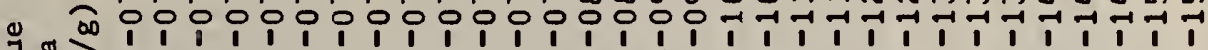
गु E 出造?

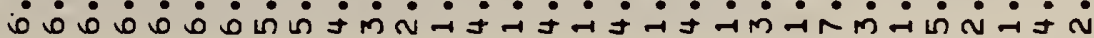

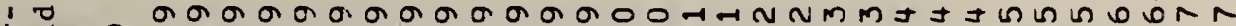
出 。

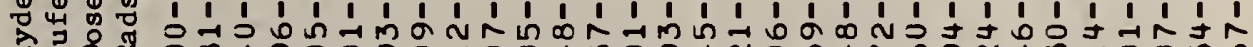

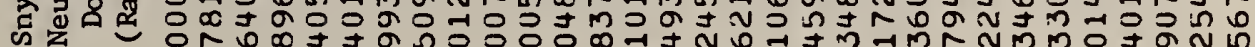

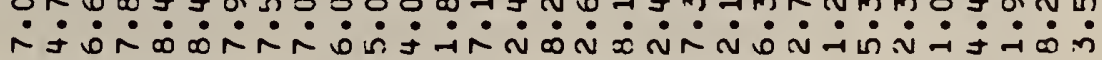

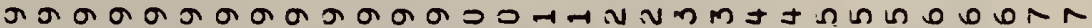

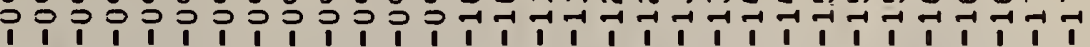

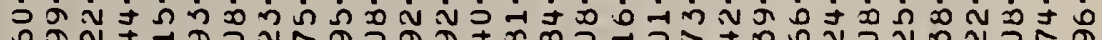

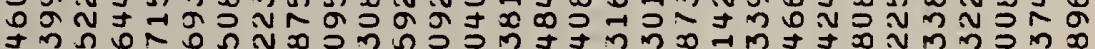

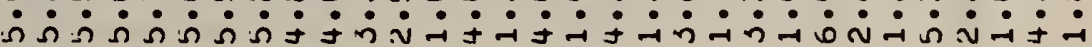

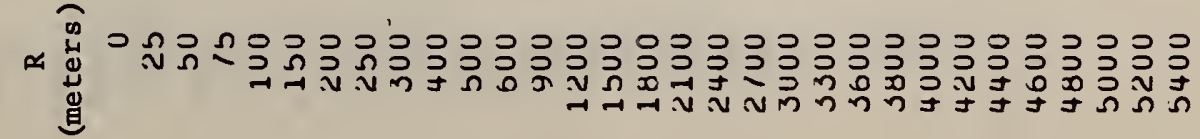


ப- 山ै

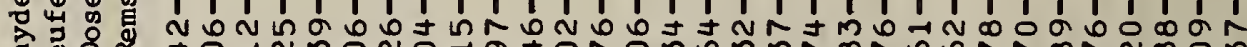

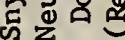
JOANMONO-1

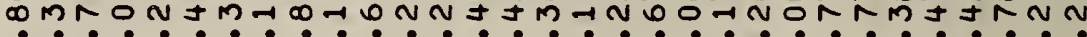

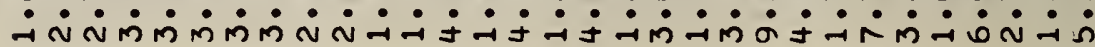

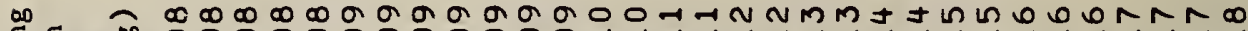

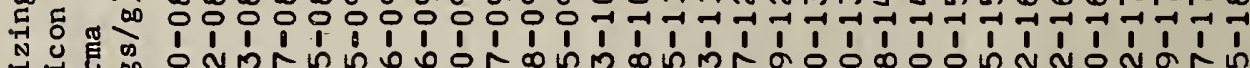
-

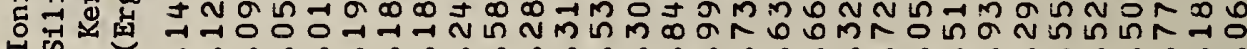

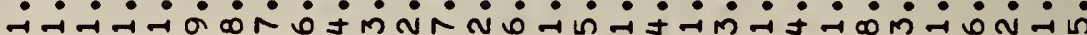

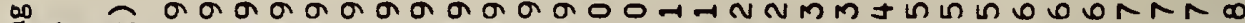

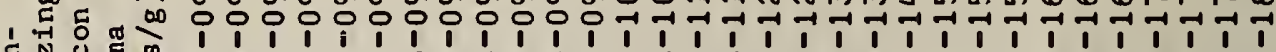

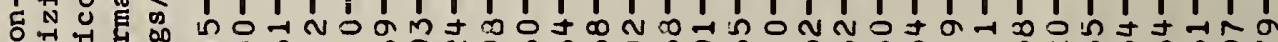
Z

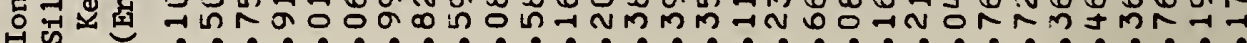

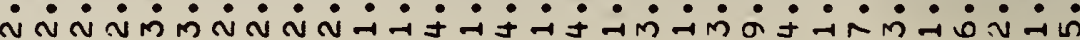

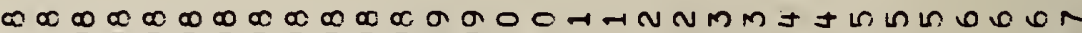

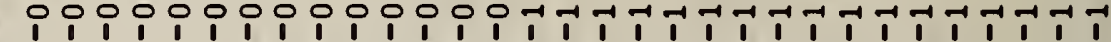

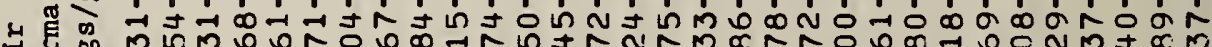
पद山

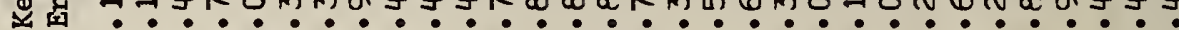

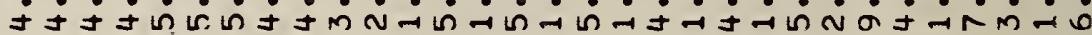

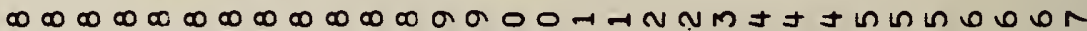

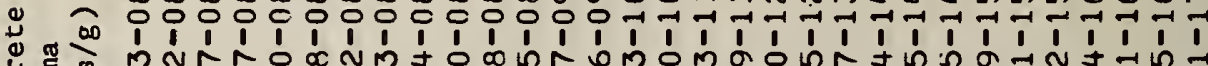
म्ञ y

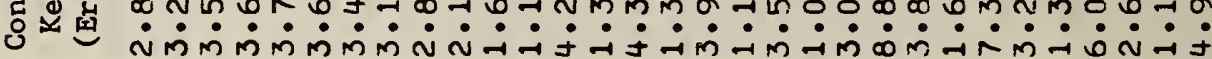

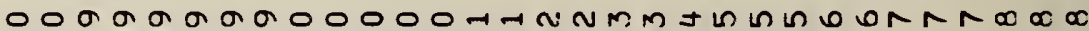

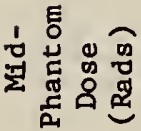

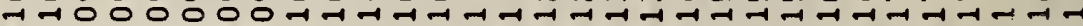

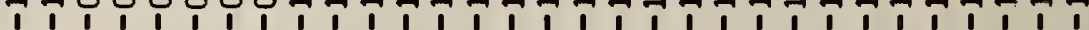

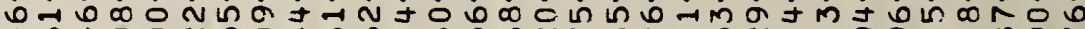

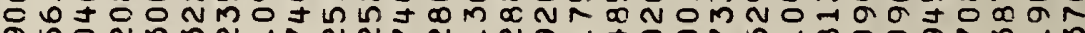

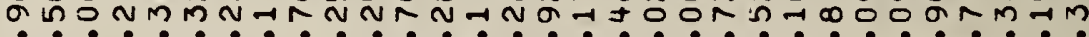

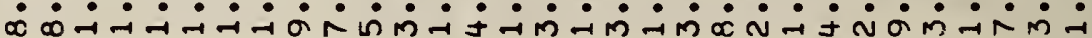

NRNANRNANR⿻ ฯ

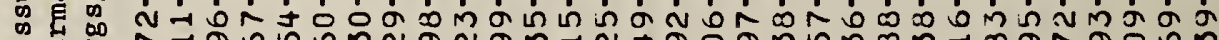

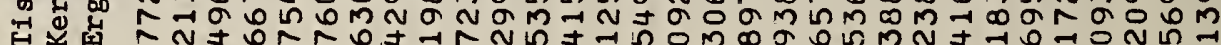

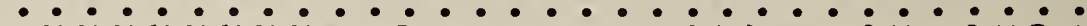

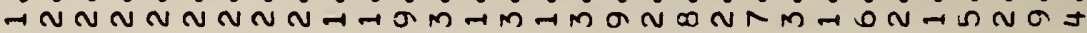

\section{究 ชै ¿}

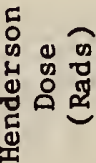

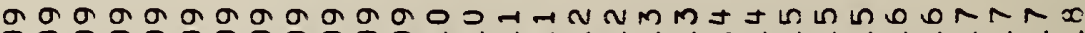
إ momojN ด I0 0 in 0 a majmara

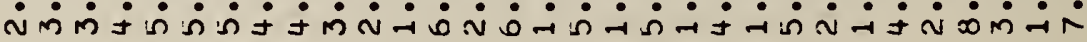

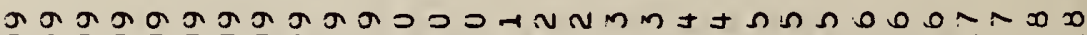

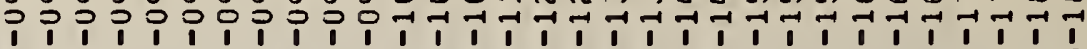

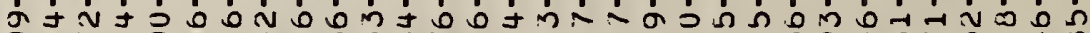
小土龰-

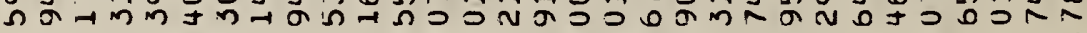

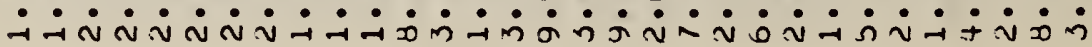

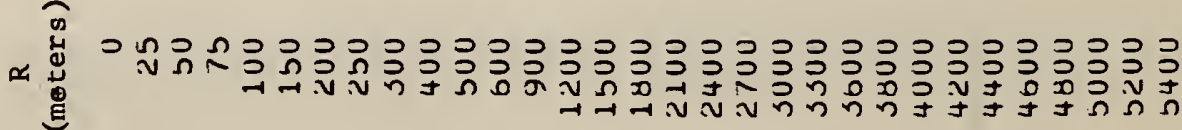




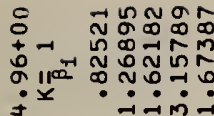

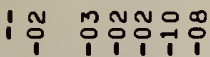

- I I 11 壬

कृष

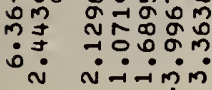

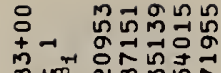

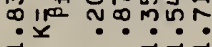

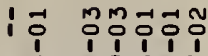

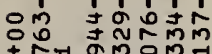

फे

?⿻

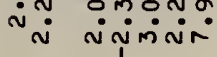

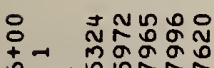

"ilon in mân

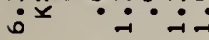

I' J 0 응

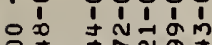

+

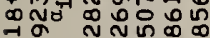

$\because N \mathbb{N}$ N

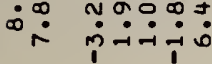

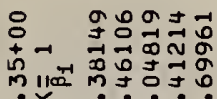
( $x$ -

|

1 $1111+1$

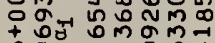

号牙察品

ก: a

$\dot{\sim} \dot{\sim} \dot{-1}-$

MNNㅁำ

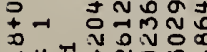

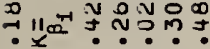

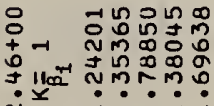

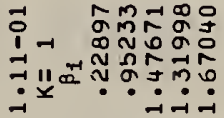

! Э ำำำ

1 0 ก

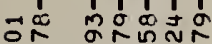

+

잉

$\Rightarrow \quad \therefore \dot{\sim} \dot{0}$

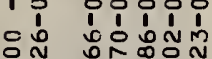

N.

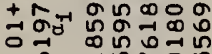

:ำ

$\because \quad$ i

| 0 헝ㅇㅇㅇㅇㅇㅇ

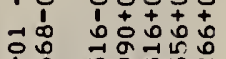

10

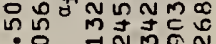

iी

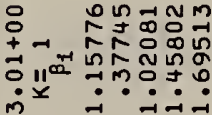

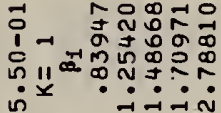

I

i gipí

오

+口 m

:-

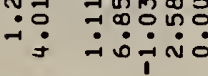

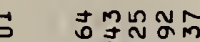

+

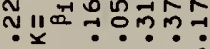

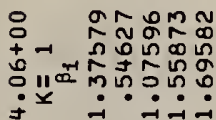

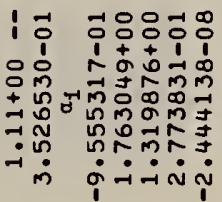

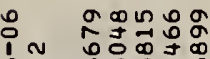

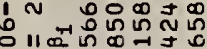

$\therefore \dot{0} \dot{0}-1$

- Nํำ

Ұ II

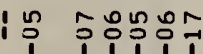

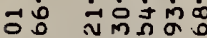

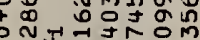

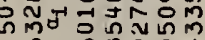

$\therefore \because$ ini

$\stackrel{\infty}{8}$

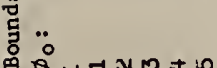

I ำ ำกำ

염

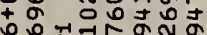

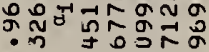

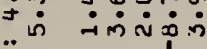

兽

| 우 유유ำ

ำ

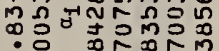

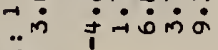

兽..

嵩

莺

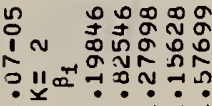

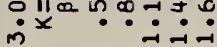

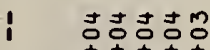

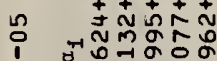

둥요

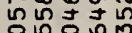

- 1 व

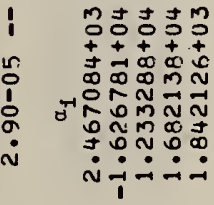

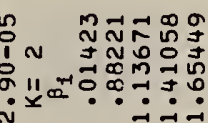

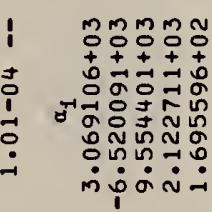

J

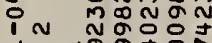

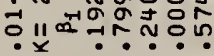

㟒

๘

के

๓๐

4.

0

ఫ

की 品

동

4 क

$\exists$

2

ํำ

$4=$

- 10

$+$

콜 궁

ฮ

000

$\rightarrow$

H.

()

os

그

(1) क

5

मी

4म क

分

(1) 0

ن-

44

(1)

की

年金

ษ 110 in

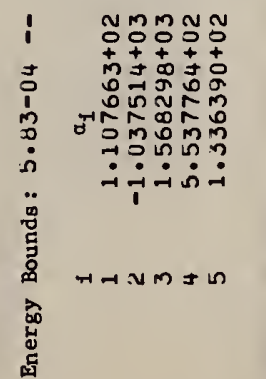

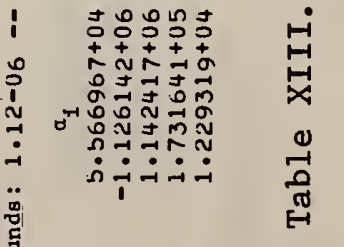

के 


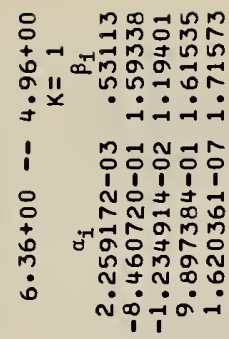

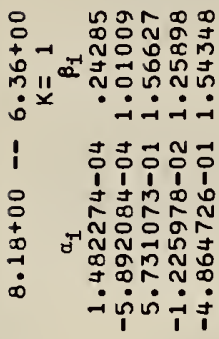

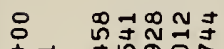

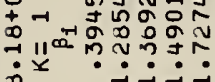

Z

월

变

$\pm$

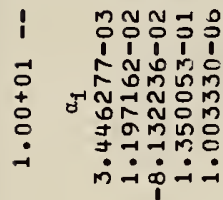

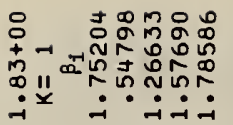

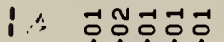

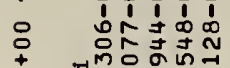

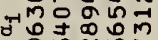

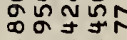

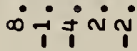

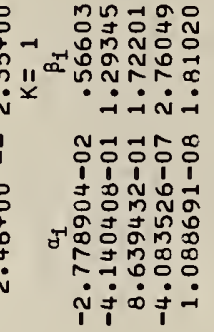

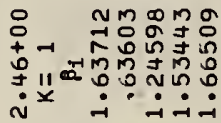

- 7 웅영

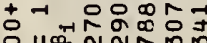

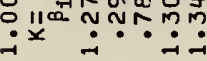

1 궁요욤

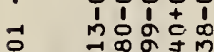

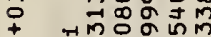

Nิ

- $\quad \dot{0} 0 \div \div$

$\overrightarrow{0}$ - م

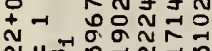

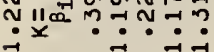

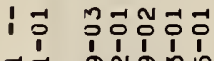

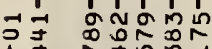

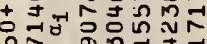

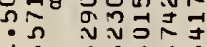

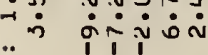

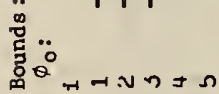

岂

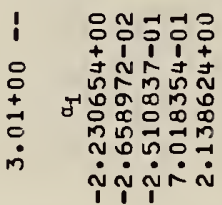

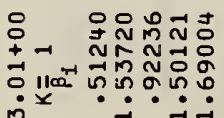

1 matô

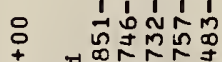

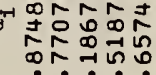

ஸिं்िं

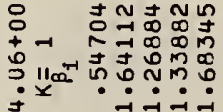

- dílí

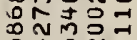

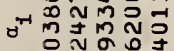

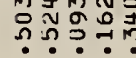

-.

哭

मัง

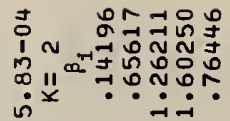

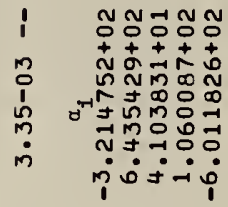

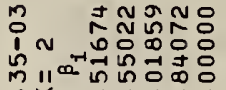

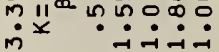

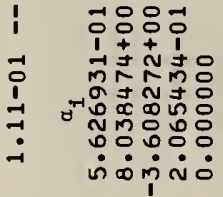

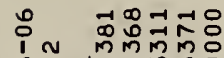

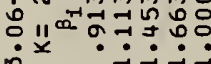

I 范吉吉

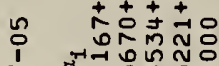

1 गี

的穴品:

ํ்

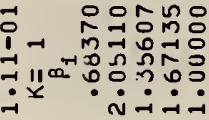

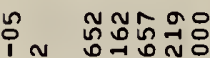
1 N

: $\ln ^{\infty}$

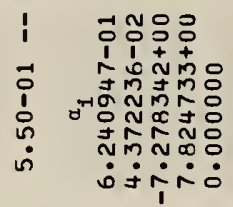

l mmot

n n

i

ㅇํำ

i

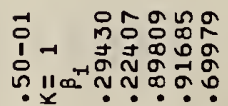

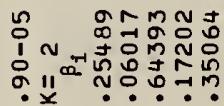

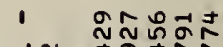

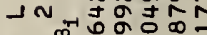

एะ

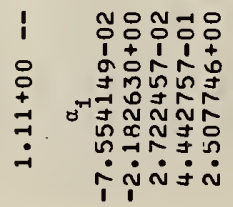

i

7mm

응ํㅇㅇㅇㅇ

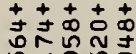

I ช

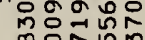

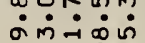

× 吉告织

山 南圭赤志

I

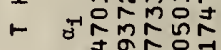

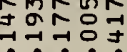

iिए内ं

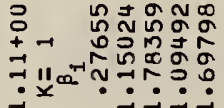

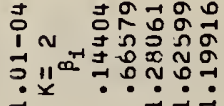

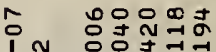

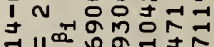

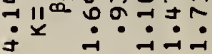

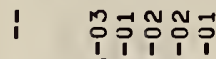

$$
\text { i }
$$

พㅇํㅇํำ

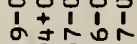

象

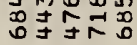

ம்

แกบกษ

แับกะก

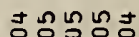

Nํㅇำกำ

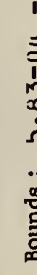

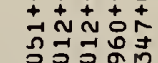

Thว

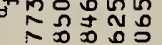

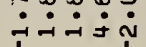

离

7

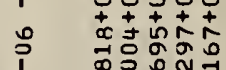

i.

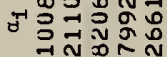

ดัง

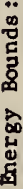

获

离

告

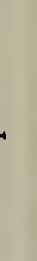




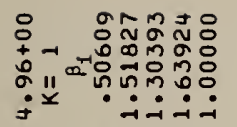

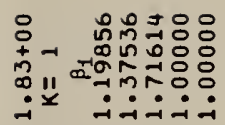

I 1 잉

I N 0 융

요요 + 0 궁ㅇㅇㅇㅇㅇ กㅇํㅁำ :?

웅 $\quad 11110$ 茴

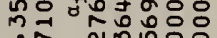
ก

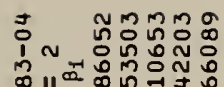
邹

I 0 옹드잉

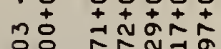
ㅇㅇำ

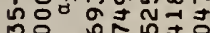

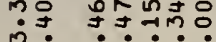
ज :

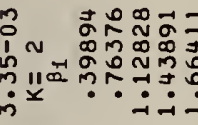

1 응 형ㅇㅇㅇㅇㅇ웅

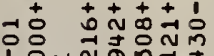

1ㅇํㅇ กี

自每

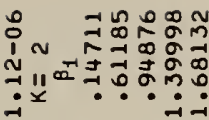

| กำํำ

के

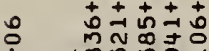

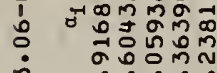

i

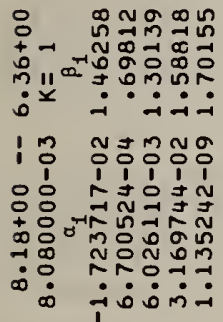

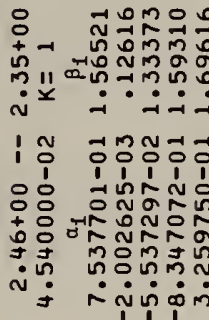

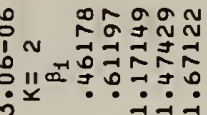

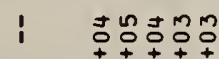

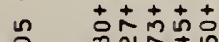

1.

กตร

i் $\dot{1} \dot{1} \dot{-}$

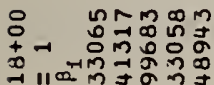

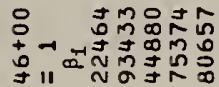

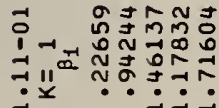

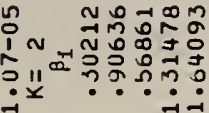

I? 영ㅇํㅇ

1 tín

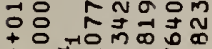

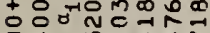

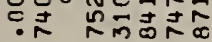

$\because \ddot{0} \dot{0} \dot{0} \dot{c}$

ix $x$ -

1 공 덩ㅎㅇㅇㅇㅇ

I ก Mำ ำ

을

+ㅇํํํํำㄴำ

m

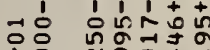

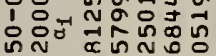

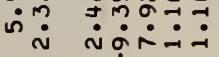

I 뭉ํㅇำ

n $+\stackrel{+}{+}+\infty+\infty$

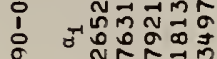

: N

ง $\because \dot{\infty} \dot{N} \dot{-1}$

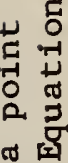

हू ญ

岂

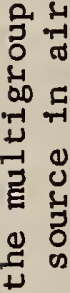

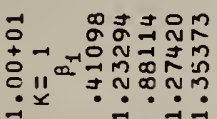

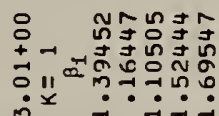

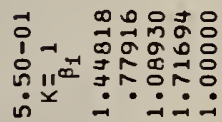

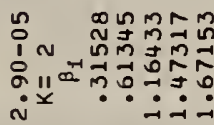

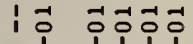

I บ ำกับ

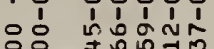

年

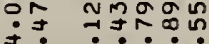

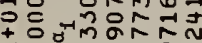

ก 0 - na

$\because \because 00 N$

ப் $\dot{0} \dot{j}$

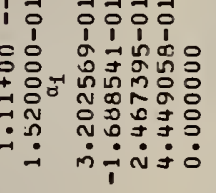

I

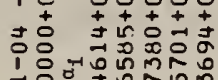

뮤

- $\dot{\sim} \dot{\sim} \dot{\sim} \dot{\sim}$

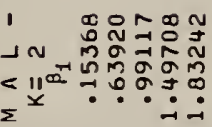

$\infty 0$

है

w

(1)

o)

कै

40

$\simeq \quad$ ก ก n

w \pm+++

I

ลี

?N $\rightarrow \Omega$

$\because \dot{0}: \dot{0}$

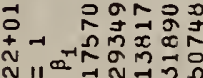

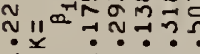

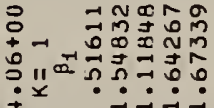

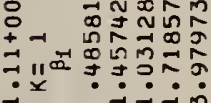

苟

: $10^{-1} \Omega 0$

- $x$ -

1 ก กีำก

$\rightarrow 0$ N $5 \infty$

I $v$ MNA

I

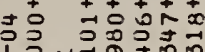

1ว

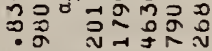

กั. บัง

总周

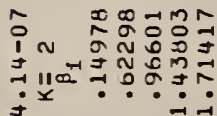

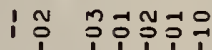

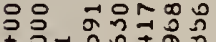

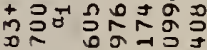

- :

离范

幽

点

กำกำำ?

圭声市市

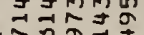

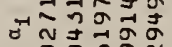

ำจำ ส

in:

잉

4 엄

क

눈

(1)

0 응

너

넌

$\begin{array}{ll}0 & 0 \\ 0 & 0 \\ 0 & 0\end{array}$

है

岕 


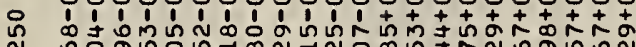

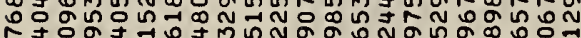
ษคம்

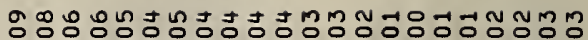

- i i i i i i i i i i i i i i o o o o o o o

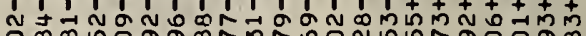

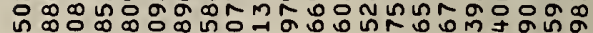

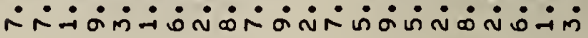

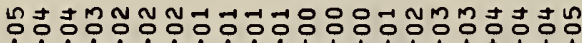

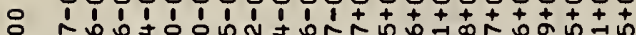

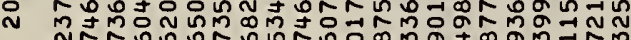
ஸேன்

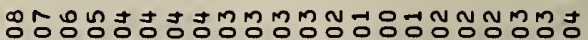
$\rightarrow \infty \rightarrow 000$ ת

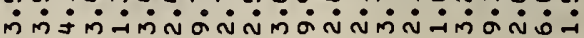

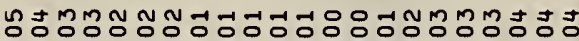

跳

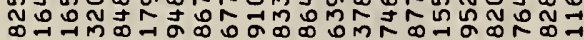

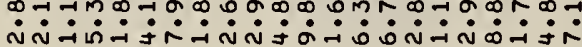

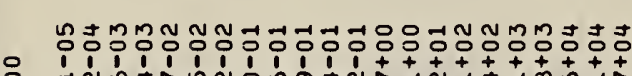

- $\rightarrow N$ N

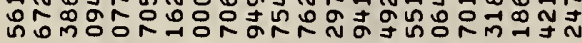

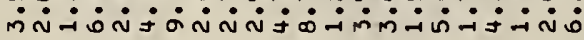

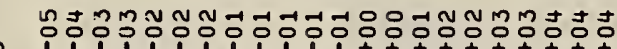

$n$

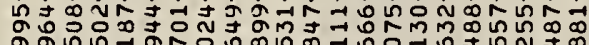

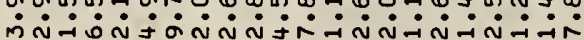

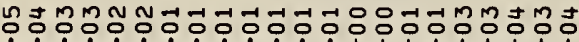

i b

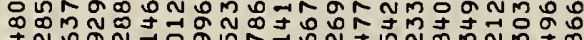

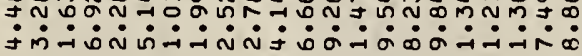

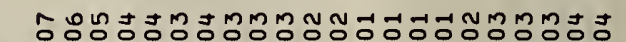

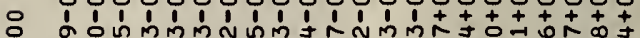
N

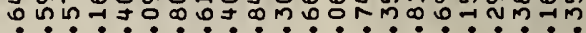

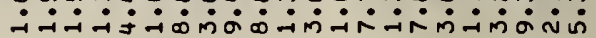

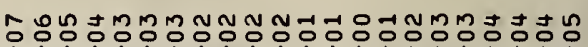

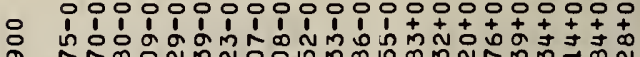

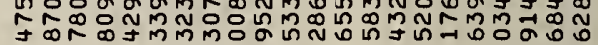

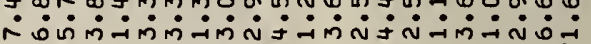

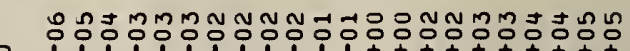

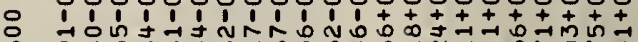

o ம்

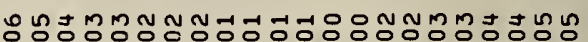

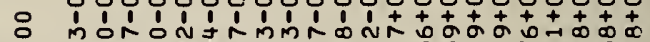

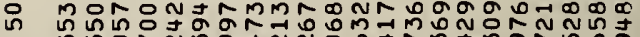
m?

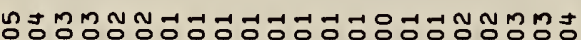

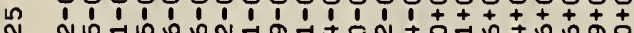
N OดศMm

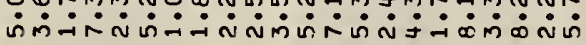

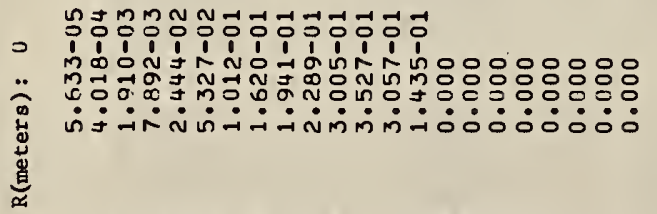

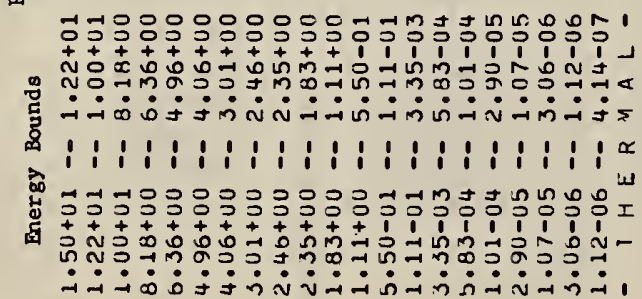

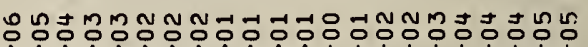

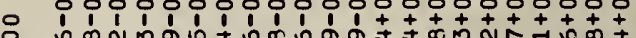

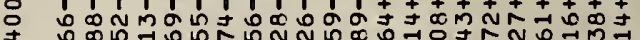

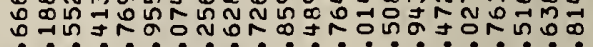

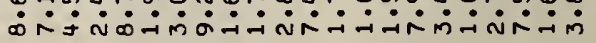

ᄂ

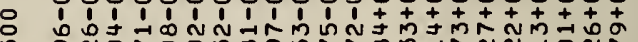
M

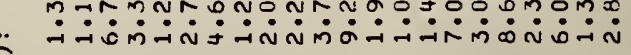
离

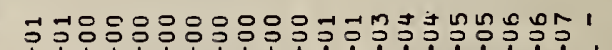

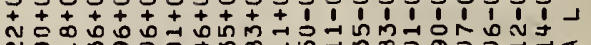

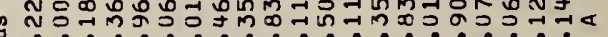

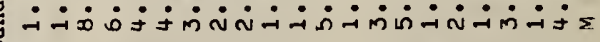

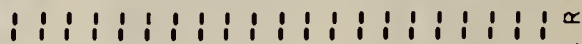

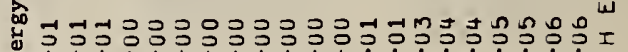

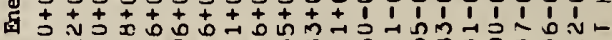

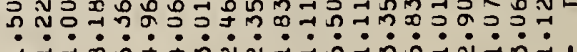

4-1

0

م्रि 


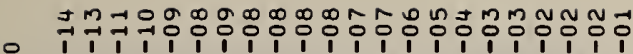

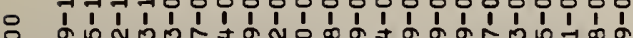

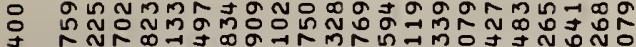
ம்

0
0
$\infty$
$m$

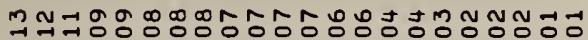

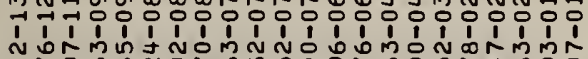

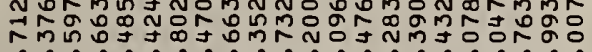
¿

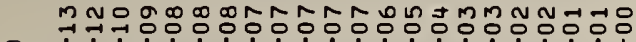

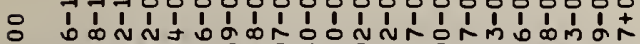

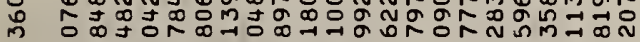

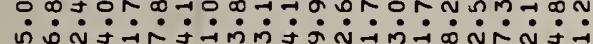

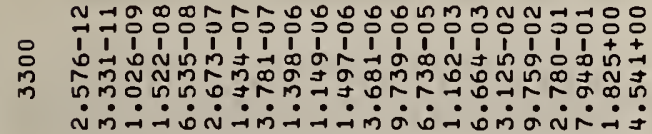

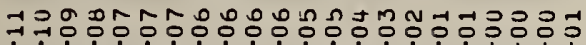

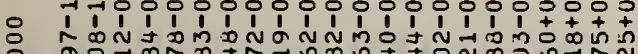
مิ

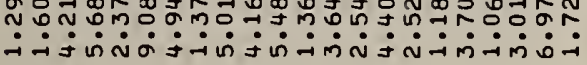
ச்

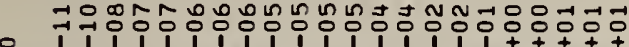

- orjád N 于

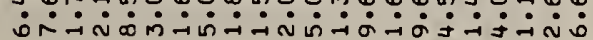

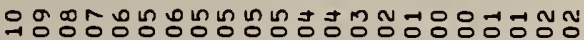

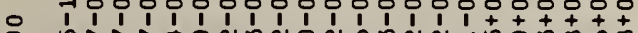

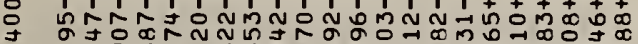

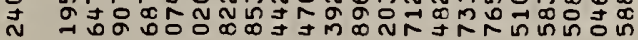

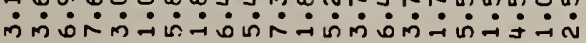

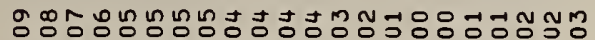

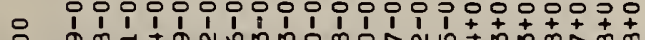
ㄷำ

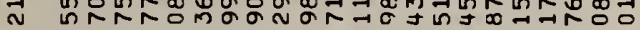

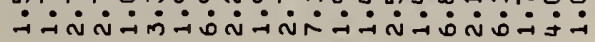

更

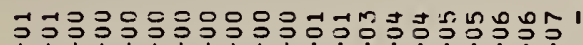

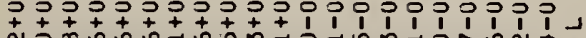

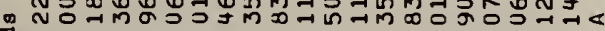

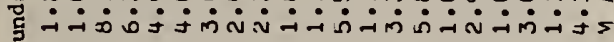

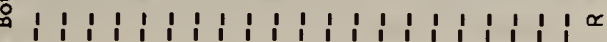
总

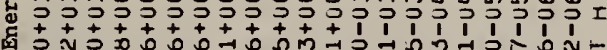

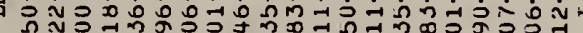
:

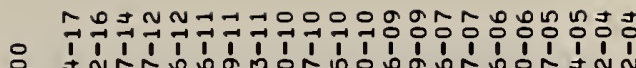

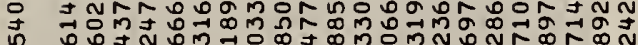

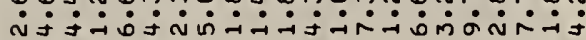

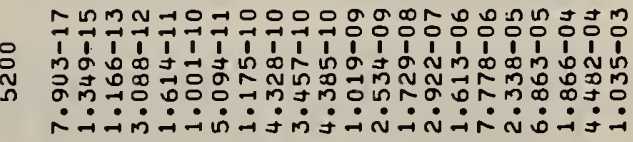

-

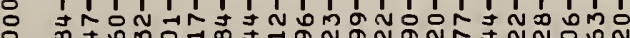

in

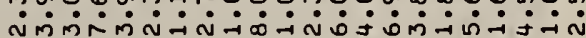

눈

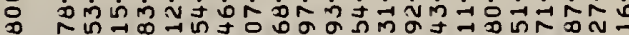

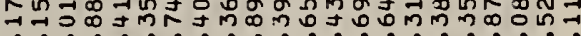

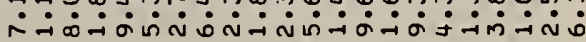

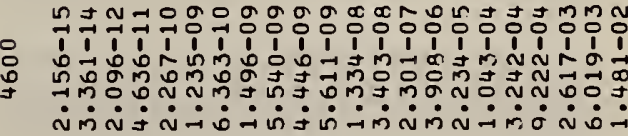

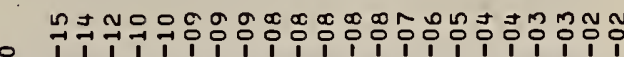

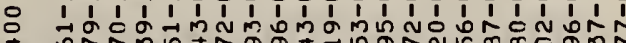

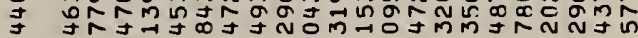

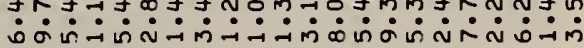

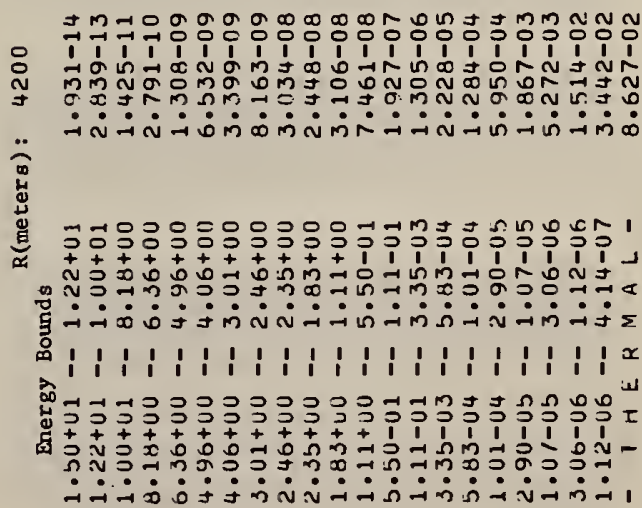




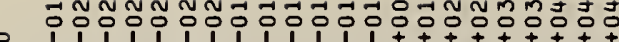

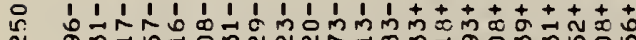

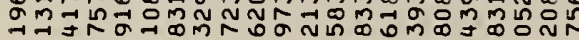

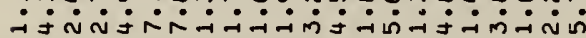

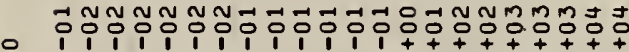

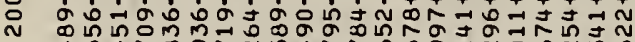

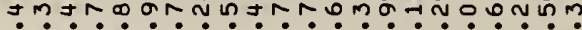
孔

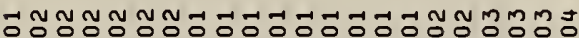

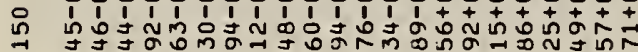
mom

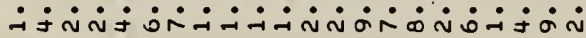

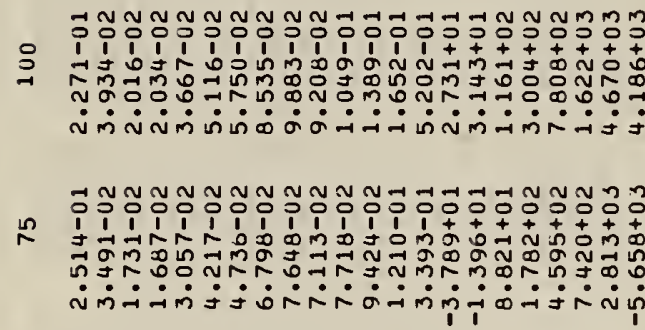

-

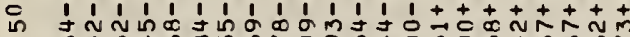

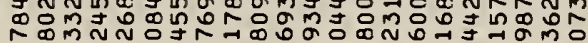

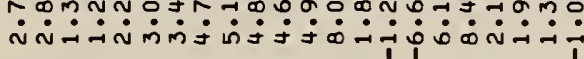

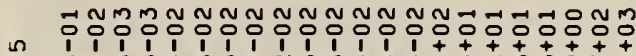
n

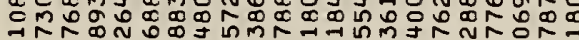

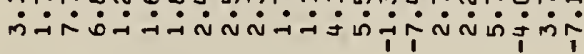

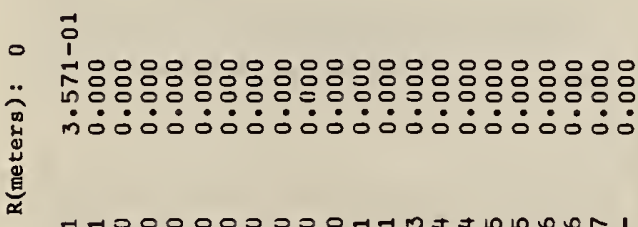

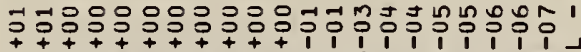

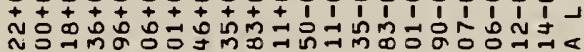

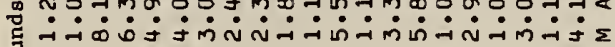

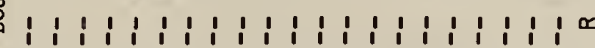

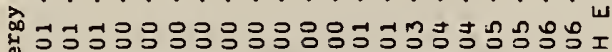

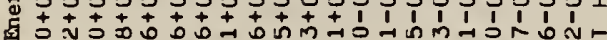

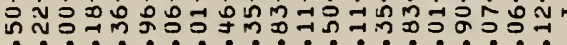

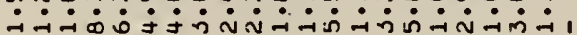

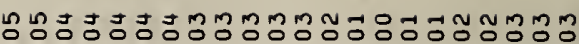
○ ธمผ

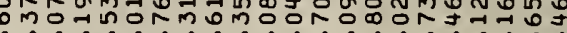

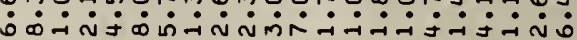

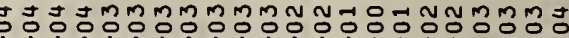

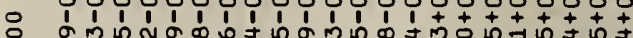

ก б

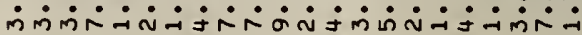

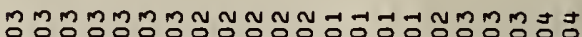

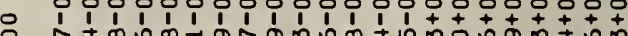

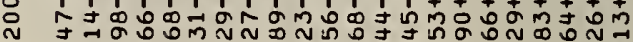

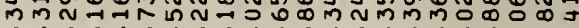

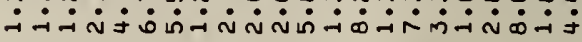

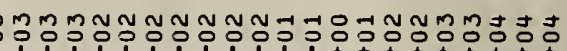

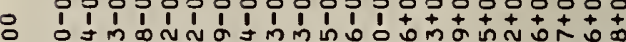

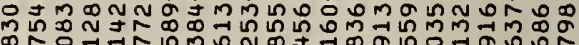

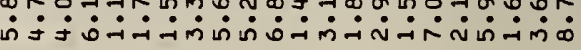

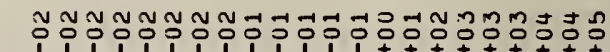

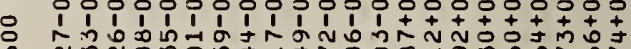

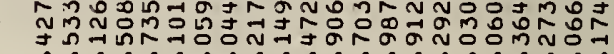

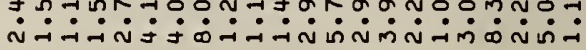

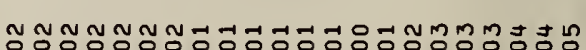

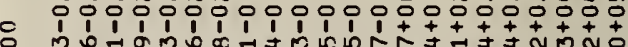

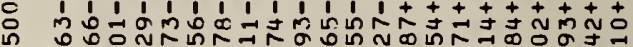

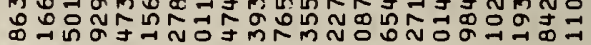

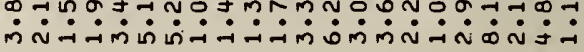

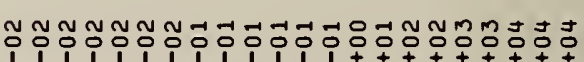

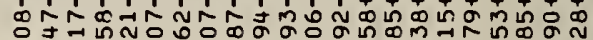
ำ大

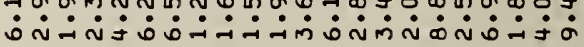

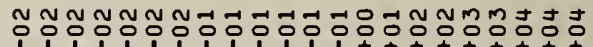

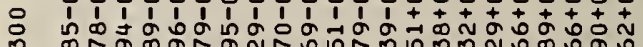

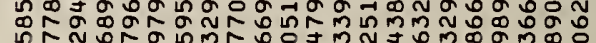

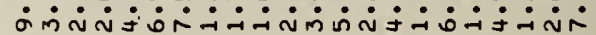

岕

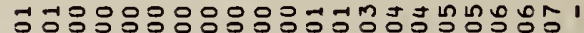

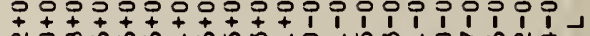
ำ 웅

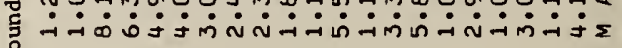

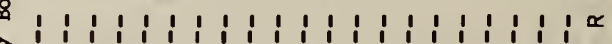
实

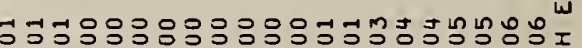

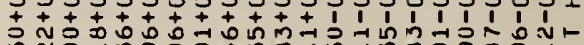
ที ำำ

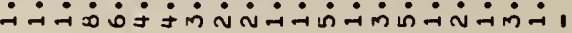




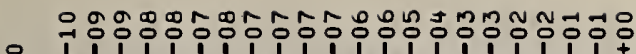

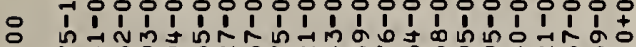
م⿻-1

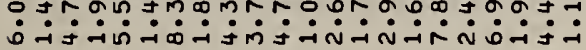

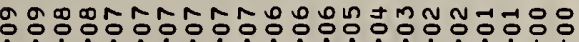
- ló

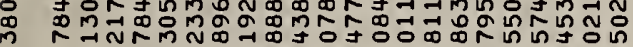

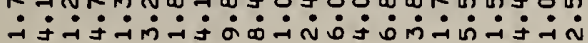

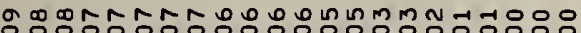

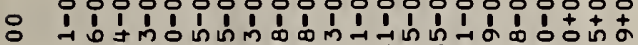

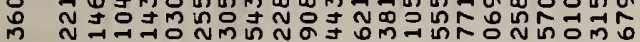

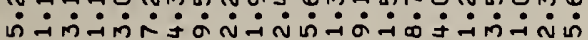

-

응

m

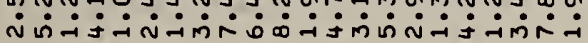

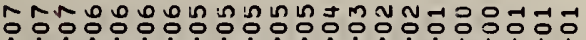

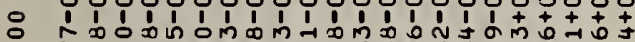

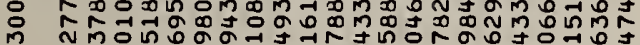

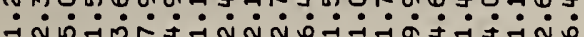

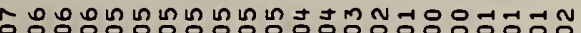

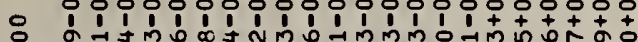

م a -1

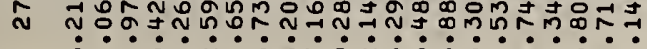

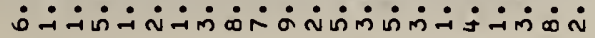

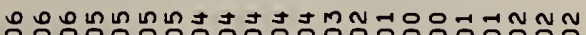

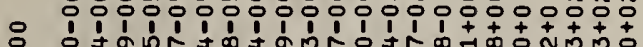

走

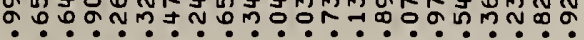

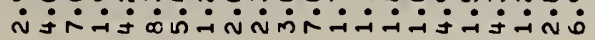

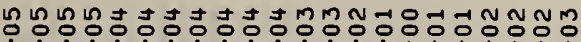

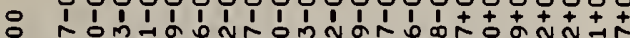

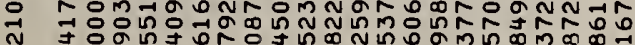

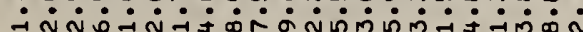

葍

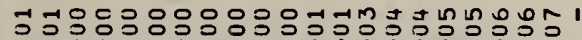

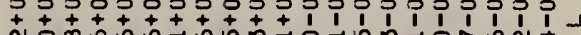

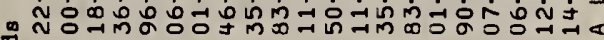
守

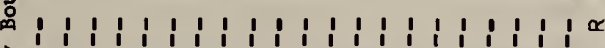
को $1-1000000000-7 m a t$ 约0

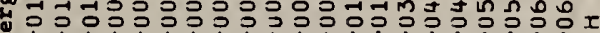

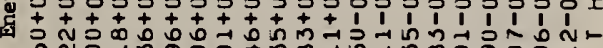

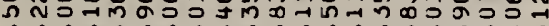

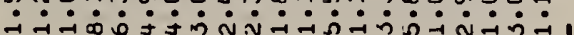

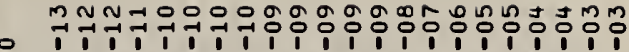

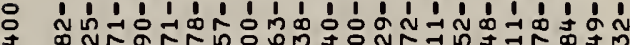

ค

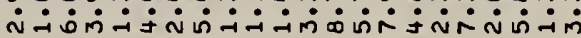

m

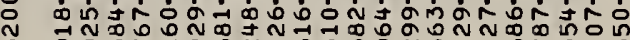

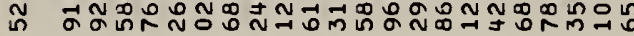

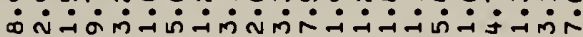

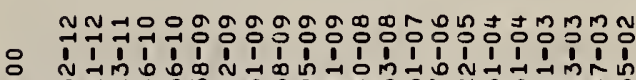

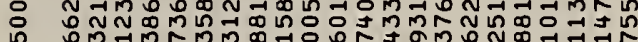
ヘ

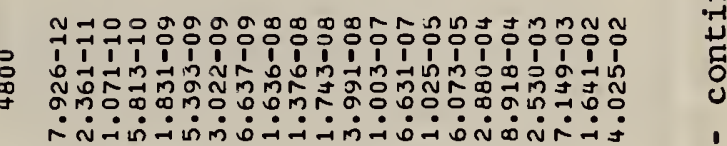

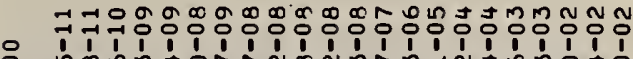

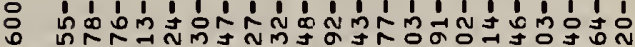

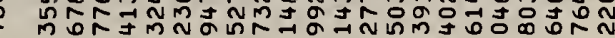

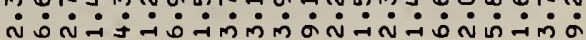

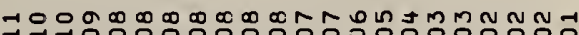
F 700000000000000

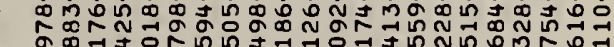

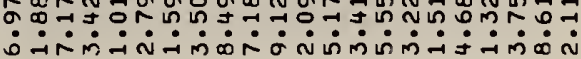

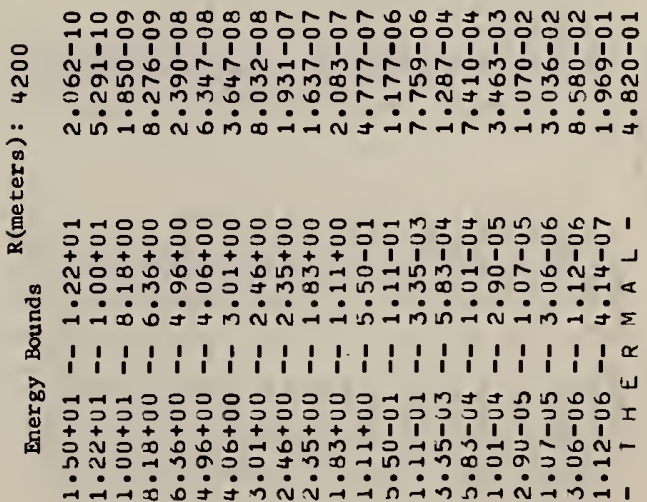




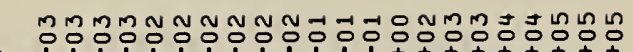

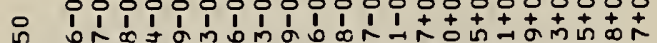

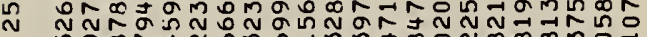

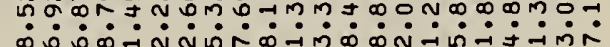

กMmM

- i i i i i i

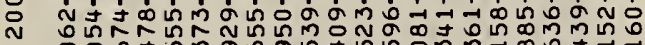
ㅇํ의

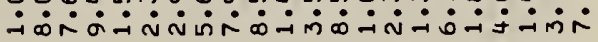

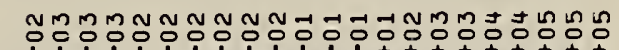

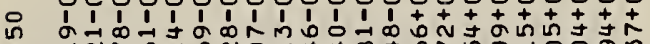

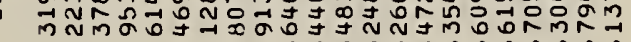

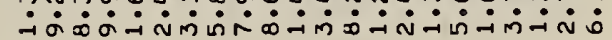

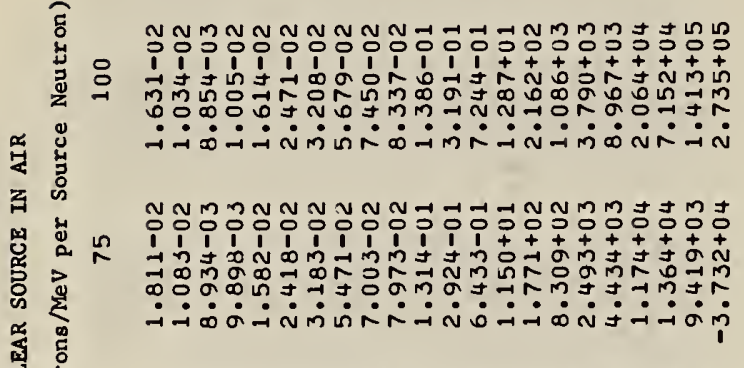

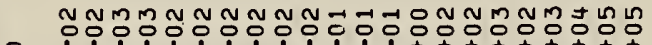
L n

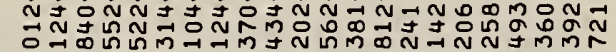

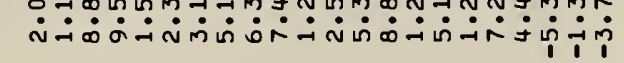
$\underset{E}{E}$

ก ก M N N N N N i

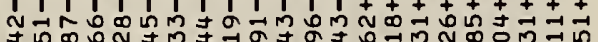

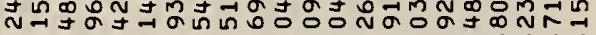

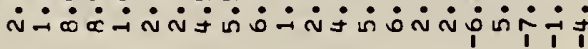

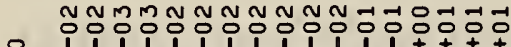

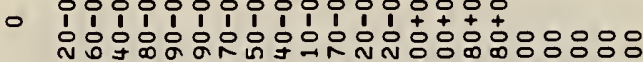

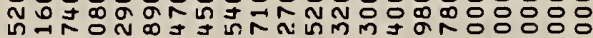

क人

峉

7-17000000000-7matan

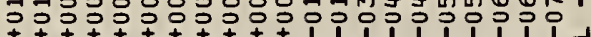

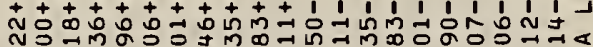

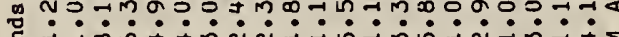

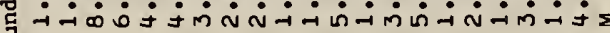

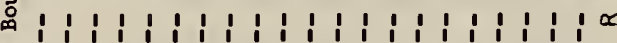

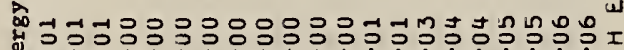

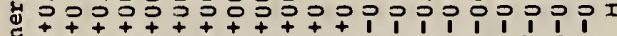

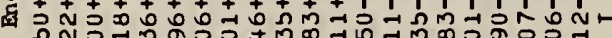

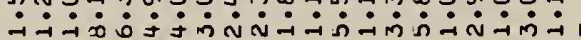

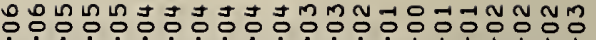

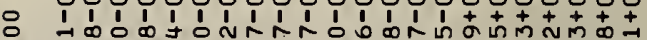

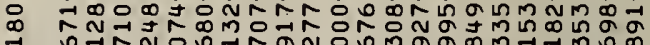

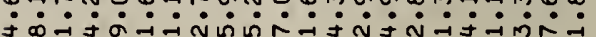

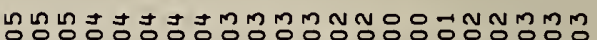

- i i i i i i i i i i i t

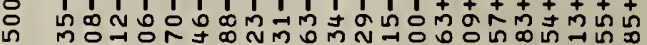
$\because$ ง

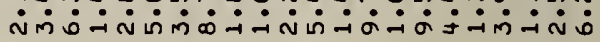

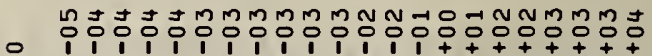

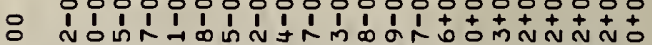

N

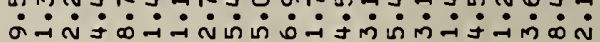

\section{¿ू}

Jีว

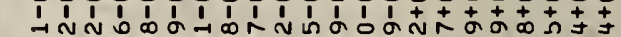

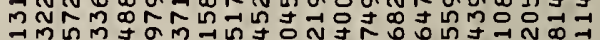

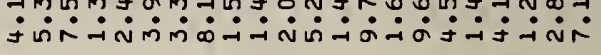

MำmmM

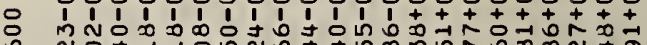

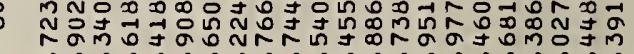

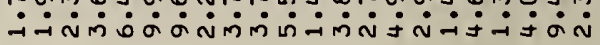

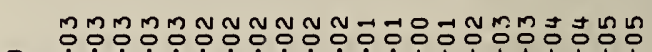

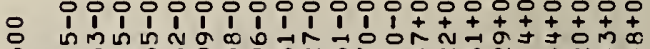

n

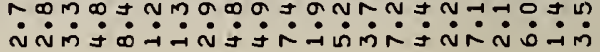

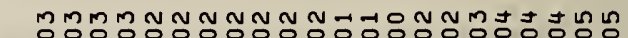

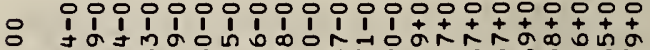

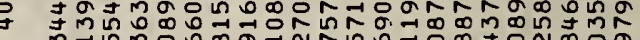

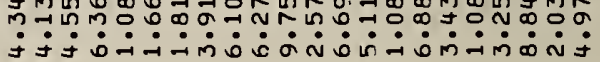

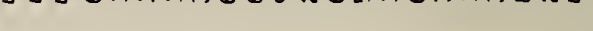

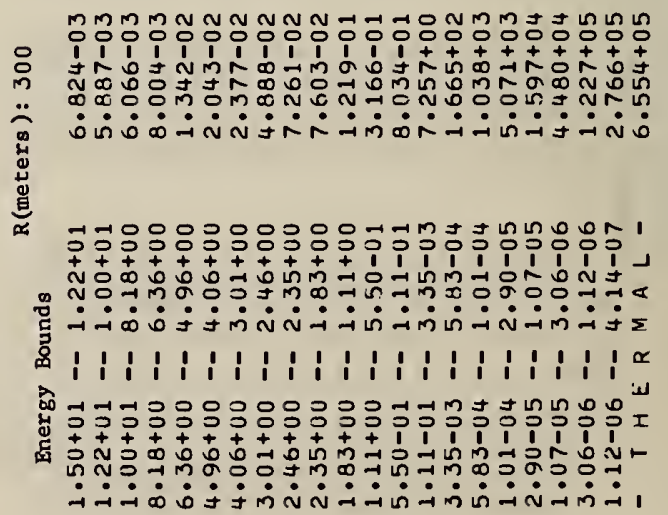




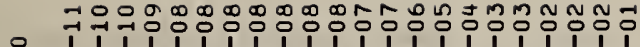

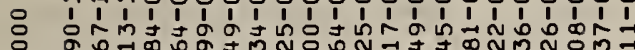

于 ษ

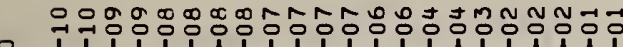

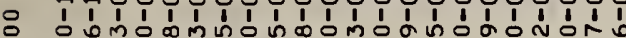

m N

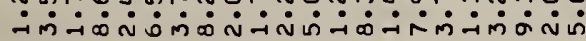

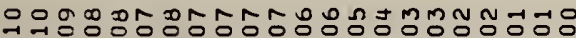

- 1

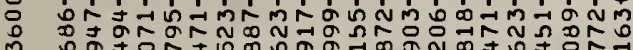

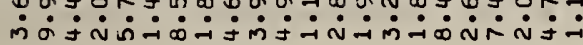

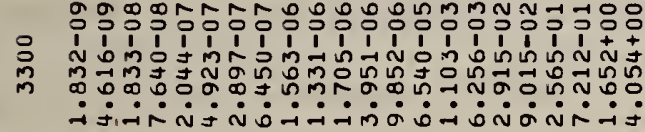

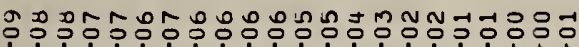

8 8 80

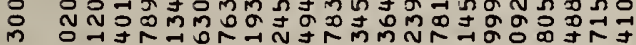

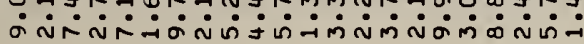

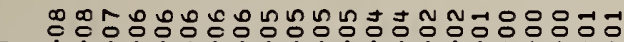

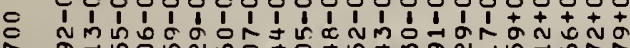

ง

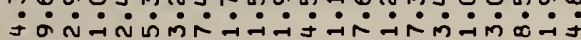

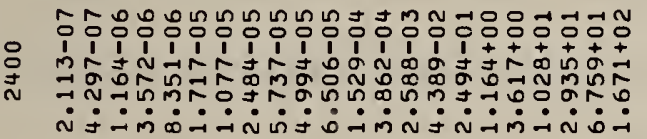

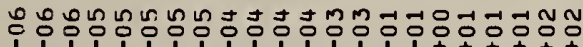

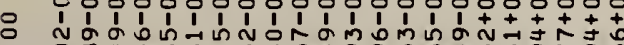

-

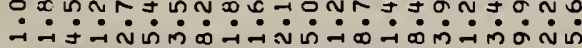
$\ddot{1}$

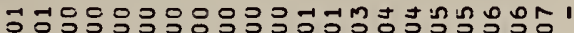

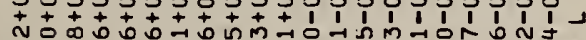

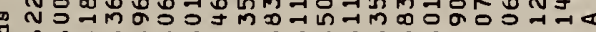

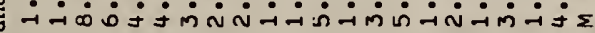
| I I i i i i i i i i i i i i i i i i i i के

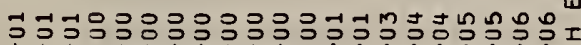

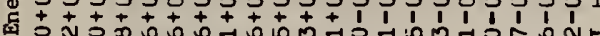

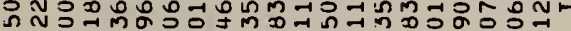

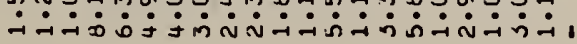

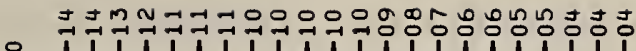

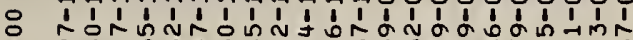

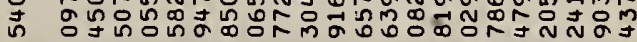

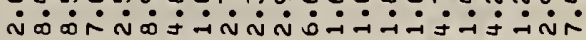

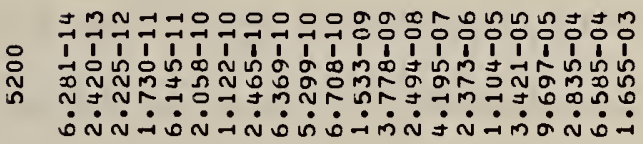

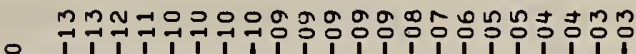

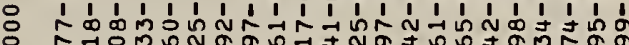

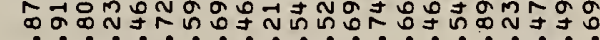

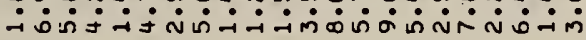

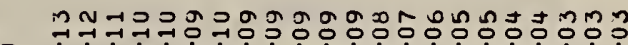

- I

0

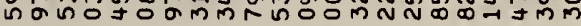

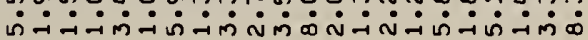

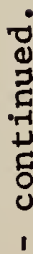

号

nN

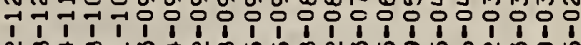

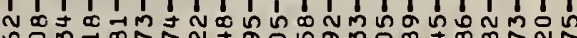

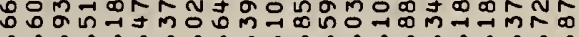

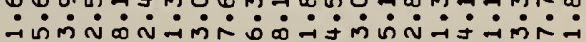

8
g
y

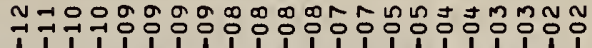

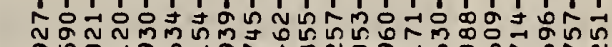

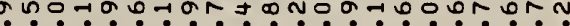

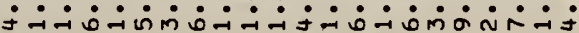

H- Ho

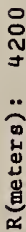
1 1 1 1 1 1 1 1 1 1 1 1 1 1 1 1 1 1 1 1 1

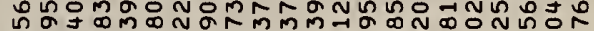
ง

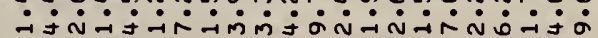

-

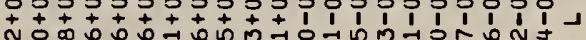

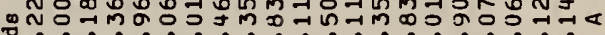

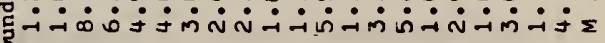

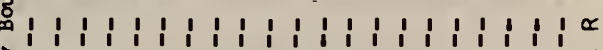

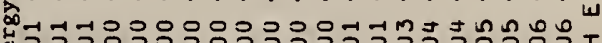

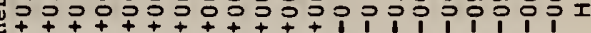

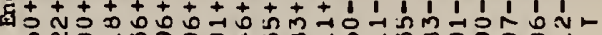
ภำำ

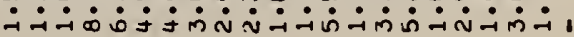


ing a Legendre sum of the harmonic coefficients for the appropriate values of $E$ and $z$. In practice, the number of coefficients available is insufficient - particularly for high energy neutrons - and spurious oscillations or even negative values occur. These oscillations can be reduced considerably by using an extrapolation technique devised by Spencer [6] for estimating harmonic coefficients for higher $l$ values. Although this technique does not eliminate oscillations, the remaining peculiarities are very of ten 1 imited to negative values of $\cos \theta$, where the angular distribution has relatively low values and is therefore of little practical interest. The angular distribution near the source, particularly for point sources, tend to exhibit trends associated with the single scattered angular distribution. Thus near the source, one expects that the angular distribution would exhibit oscillations similar to those of the scattering kernel.

In Figure 4 we show a comparison between moments and $S_{n}$ calculations of the Snyder-Neufeld dose angular distribution for a fission source. These comparisons indicate that the moment results tend to be more peaked forward than the $S_{n}$ results. Consequently more neutrons would transport further from the source. This phenomena also contributes to the higher dose values at large distances that is predicted by the moments calculations.

The authors wish to express their thanks to Dr. Lewis V. Spencer, whose encouragement and advice helped make this paper possible. 


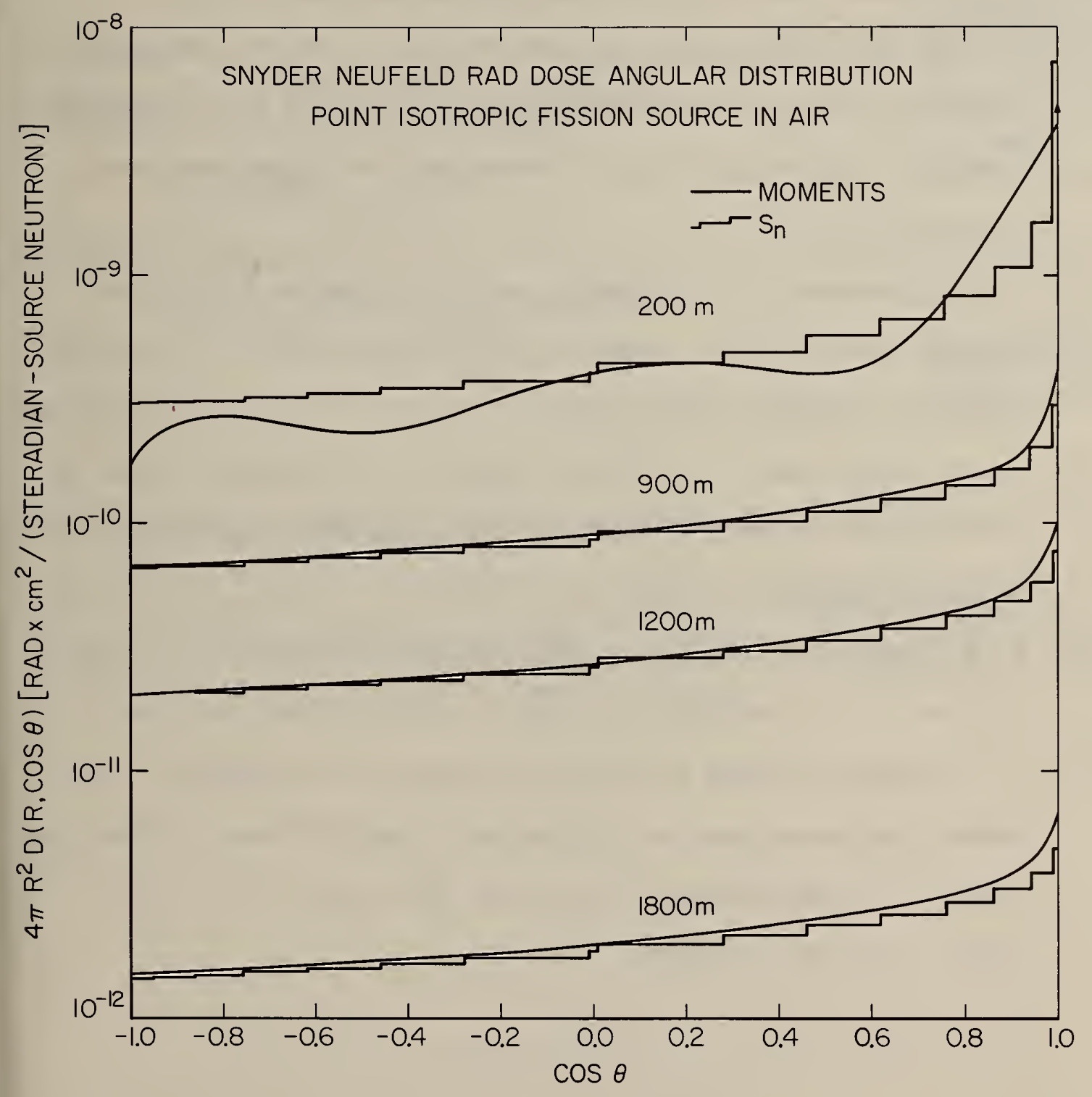

Figure 4. Comparison of moments and $S_{n}$ (Ref. 5) calculations of the angular distribution of Snyder-Neufeld dose in rads at four depths in air. 


\section{REFERENCES}

[1] J. T. Reynolds and E. L. Slaggie, KAPL-M-6452 (1965).

[2] C. W. Craven, Oak Ridge National Laboratory (June 1966).

[3] E. A.Straker, Shielding Benchmark Problem 3.0 Neutron Spectrum from Point Fission and $14 \mathrm{MeV}$ Sources in Infinite Air, ORNL-RSIC(ANS-SD-9) Oak Ridge National Laboratory, Oak Ridge, Tennessee (August 1970).

[4] C. M. Ei senhauer, G. L. Simmons, and L. V. Spencer, A Neutron Moments Computer Code, Moment I, NBS Technical Note 725, National Bureau of Standards (May 1972).

[5] E. A. Straker and M. L. Gritzner, Neutron and Secondary Gamma Ray Transport in Infinite Homogeneous Air, ORNL-4464, Oak Ridge National Laboratory (1969).

[6] L. V. Spencer, Plural Series Representation of Functions, to be published in J. Res. Nat. Bur. Stand. (U.S.) (Phys. and Chem.).

[7] L. V. Spencer, Bounds to Truncation Errors in Biorthogonal Polynomial Approximations, with Illustrative Applications to Gamma Ray Transport Distributions, J. Res. Nat. Bur. Stand. (U.S.) 76B, (Math. Sci.) No. 1 (1971). 
FORM NBS-114A (1-71)

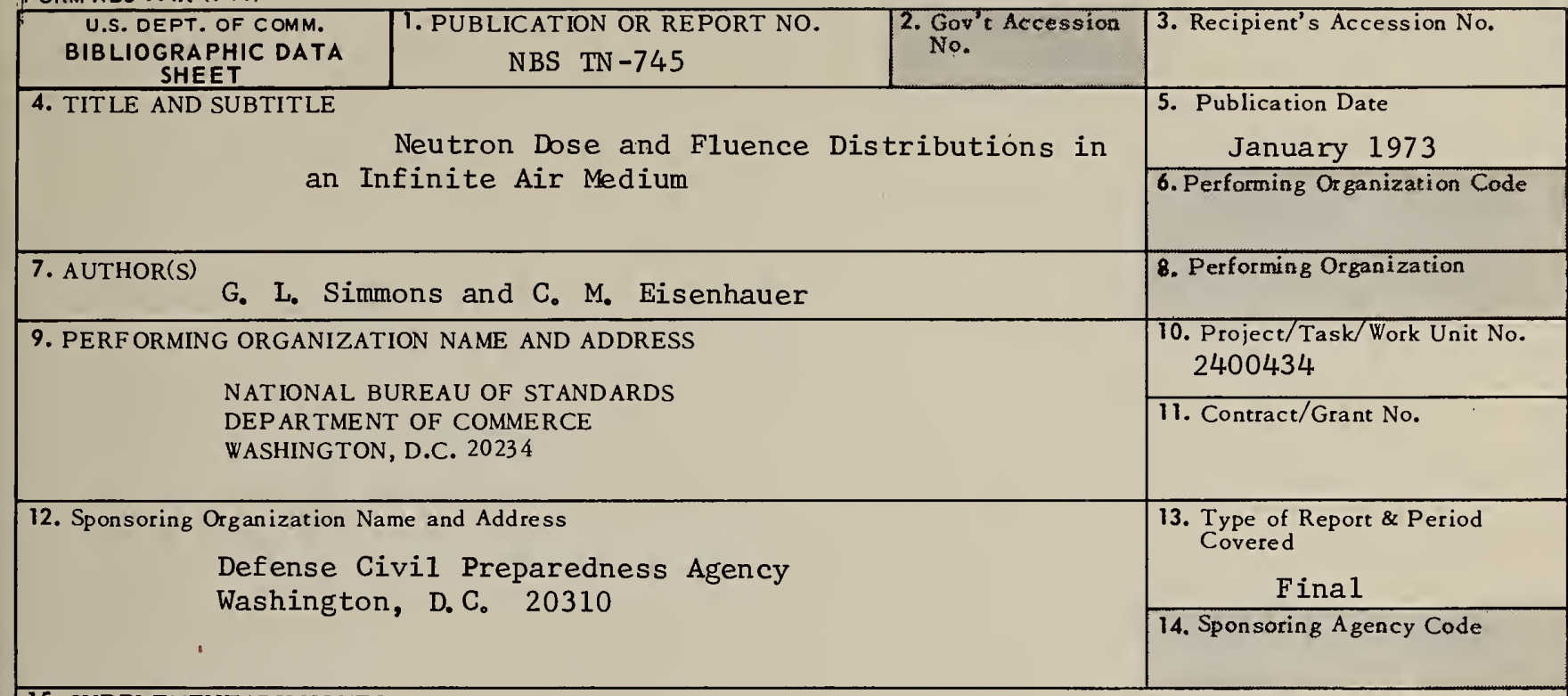

\section{SUPPLEMENTARY NOTES}

16. ABSTRACT (A 200-word or less factual summary of most significant information. If document includes a significant bibliography or literature survey, mention it here.)

The moments method is applied to the problem of calculating the neutron dose and fluence distributions in an infinite medium of air. These calculations are compared with Monte Carlo and Discrete Ordinates $\left(S_{n}\right)$ results. Simple parametric representations for the distributions are given which facilitate the calculation of dose and flux distributions in air with a different density.

\section{KEY WORDS (Alphabetical order, separated by semicolons) Benchmark problems; dose distributions;} moments method; neutron penetration; shielding; weapons radiation.

18. AVAILABILITY STATEMENT

$\mathrm{x}$ UNL IMIT ED.

$\square$ FOR OFFICIAL DISTRIBUTION. DO NOT RELEASE TO NTIS.

\begin{tabular}{|l|l|}
\hline $\begin{array}{c}\text { 19. SECURITY CLASS } \\
\text { (THIS REPORT) } \\
\text { X }\end{array}$ & 21. NO. OF PAGES \\
UNCL ASSIFIED & 39 \\
\hline $\begin{array}{l}\text { 20. SECURITY CLASS } \\
\text { (THIS PAGE) }\end{array}$ & $\begin{array}{l}\text { 22. Price } \\
\text { \$.65 Domestic Postpaid } \\
\text { \$N.45 GPO Bookstore }\end{array}$ \\
\hline
\end{tabular}

USCOMM-DC $66244-P 71$ 

U.S. DEPARTMENT OF COMMERCE

National Bureau of Standards

Washington. D.C. 20234

OFFICIAL BUSINESS

Penalty for Private Use, $\$ 300$
POSTAGE AND FEES PAID U.S. DEPARTMENT OF COMMERCE 